\title{
ON RANDOM FOURIER SERIES ${ }^{1}$
}

BY

\author{
JACK CUZICK AND TZE LEUNG LAI
}

\begin{abstract}
Motivated by Riemann's $R_{1}$ summation method for i.i.d. random variables $X_{1}, X_{2}, \ldots$, this paper studies random Fourier series of the form $\Sigma_{1}^{\infty} a_{n} X_{n} \sin \left(n t+\Phi_{n}\right)$, where $\left\{a_{n}\right\}$ is a sequence of constants and $\left\{\Phi_{n}\right\}$ is a sequence of independent random variables which are independent of $\left\{X_{n}\right\}$. Questions of continuity and of unboundedness are analyzed through the interplay between the asymptotic properties of $\left\{a_{n}\right\}$ and the tail distribution of $X_{1}$. A law of the iterated logarithm for the local behavior of the series is also obtained and extends the classical result for Brownian motion to a general class of random Fourier series.
\end{abstract}

1. Introduction and summary. In this paper we study random Fourier series of the form

$$
S(t)=\sum_{n=1}^{\infty} a_{n} X_{n} \sin \left(n t+\Phi_{n}\right)
$$

where $\left\{a_{n}\right\}$ is a sequence of constants, $X, X_{1}, X_{2}, \ldots$ are independent and identically distributed (i.i.d.) random variables, and $\left\{\Phi_{n}\right\}$ is a sequence of independent random variables (possibly constants) which are also independent of the sequence $\left\{X_{n}\right\}$. These notations and assumptions will be used throughout the sequel. Classical examples of (1.1) are the Rademacher series (where the $X_{n}$ take the values 1 and -1 with probability $\frac{1}{2}$ and the $\Phi_{n}$ are constants) and the Steinhaus series (where the

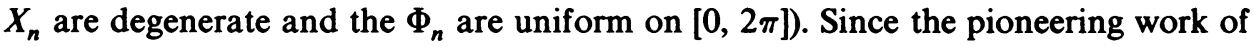
Paley and Zygmund [23] on the Rademacher and Steinhaus series, a great deal has been learned about the analytic properties (such as pointwise convergence, continuity, etc.) of these series, and the results have been extended to the general setting in which $X_{n}$ are independent, symmetric (or more generally $X_{n} e^{i \Phi_{n}}$ are symmetric), and have finite variances but are not necessarily identically distributed (cf. [10], [20], [21]).

In $\$ \S 2$ and 3 we shall extend the classical results on the Rademacher and Steinhaus series in another direction. We shall assume that the $X_{n}$ are i.i.d. but drop the usual assumptions of finite variance and symmetry. For every nonnegative function $f$ on $[0,2 \pi]$, let $\bar{f}$ denote the nondecreasing rearrangement of $f$, i.e., $\bar{f}(x)=\sup \{y: \lambda\{t \in[0,2 \pi]: f(t)<y\}<x\}$ for $0<x<2 \pi$, where $\lambda$ denotes Lebesgue measure. Let $M(f(x))=\bar{f}(x)$. This notation will be used throughout the

Received by the editors January 11, 1979.

AMS (MOS) subject classifications (1970). Primary 60F15, 60G17; Secondary $42 \mathrm{~A} 16$.

Key words and phrases. Riemann's $R_{1}$ summability, convergence, continuity, unboundedness, law of the iterated logarithm, random Fourier series.

${ }^{1}$ Research supported by the National Science Foundation under grant NSF-MCS-78-09179. 
sequel. Note that in the present i.i.d. case, if $X$ is symmetric and has a finite variance $\sigma^{2}$, then for every sequence $\left\{a_{n}\right\} \in l^{2}, S(t)$ converges a.s. for every $t$; moreover, assuming that $\sigma \neq 0$, a necessary and sufficient condition for the a.s. uniform convergence of $S(\cdot)$ is

$$
\int_{0}^{\delta}\left[M\left(\left\{\sum_{n=1}^{\infty} a_{n}^{2} \sin ^{2} \frac{n t}{2}\right\}^{1 / 2}\right) /\left\{t|\log t|^{1 / 2}\right\}\right] d t<\infty
$$

$$
\text { for some } 0<\delta<1
$$

(see Corollary 2.8 of [20]). However, when $E X^{2}=\infty,(1.2)$ is no longer sufficient for the a.s. uniform convergence of $S(\cdot)$, and in fact (1.2) does not even guarantee that the series (1.1) converges a.s. for every fixed $t$. On the other hand, if $X$ is nondegenerate, the condition (1.2) is still necessary for the a.s. uniform convergence of $S(\cdot)$ even when $E X^{2}=\infty$ or when $X$ is not symmetric (see $\$ 2$ below). For a given sequence $\left\{a_{n}\right\}$ satisfying (1.2), it is therefore natural to ask what minimal conditions on the tail distribution of $X$ will guarantee the a.s. uniform convergence of $S(\cdot)$. We shall study this problem in $\$ \S 2$ and 3 . To give some flavor of the interplay between the sequence $\left\{a_{n}\right\}$ and the tail distribution of $X$ in the conditions for the a.s. uniform convergence of $S(\cdot)$, we now specialize the general results of $\S \S 2$ and 3 to the particular case $a_{n}=n^{-\alpha}\left(\alpha>\frac{1}{2}\right)$ in the following

THEOREM 1. Let $\alpha>\frac{1}{2}$, and set $a_{n}=n^{-\alpha}$ in (1.1).

(i) If $E|X|^{1 / \alpha}<\infty$, then $P[S(t)$ converges a.e. $]=1$, where "a.e." (almost everywhere) is with respect to Lebesgue measure. Moreover, if $\Phi_{n}=0$ for all $n$, then

$$
S(t) \text { converges a.s. for every fixed } t \Leftrightarrow E|X|^{1 / \alpha}<\infty \text {. }
$$

(ii) For $\alpha>1$, if $E|X|^{1 / \alpha}<\infty$, then

$$
P[S(t) \text { converges uniformly in } t]=1 \text {. }
$$

(iii) For $\frac{1}{2}<\alpha<1$, if $E X=0$ and $E\left\{|X|^{1 / \alpha}\left(\log ^{+}|X|\right)^{\beta}\right\}<\infty$ for some $\beta>$ $(1-\alpha) / \alpha$, then (1.4) still holds.

(iv) For $\alpha=1$, if $E X=0$ and $E(|X| \log |X|)<\infty$, then (1.4) still holds.

(v) For $\alpha=1$, if either $X$ is symmetric or $e^{i \Phi_{n}}$ is symmetric for all $n$, then (1.4) still holds under the weaker moment condition that $E\{|X| \log \log (|X|+e)\}<\infty$.

(vi) For $\frac{1}{2}<\alpha<1$, there exists a symmetric random variable $X$ such that $E\left\{|X|^{1 / \alpha}\left(\log ^{+}|X|\right)^{\beta}\right\}<\infty$ for all $\beta<(1-\alpha) / \alpha$ and

$$
P[S(t) \text { converges at every } t]=0
$$

for all choices of the marginal distributions of $\left\{\Phi_{n}\right\}$.

(vii) For $\alpha=1$, there exists a symmetric random variable $X$ such that $E\left\{|X|[\log \log (|X|+e)]^{\beta}\right\}<\infty$ for all $\beta<1$ and (1.5) holds for all choices of $\left\{\Phi_{n}\right\}$. Moreover, there exists a zero-mean random variable $X$ and a sequence $\left\{\Phi_{n}\right\}$ such that (1.5) holds and $E\left\{|X|\left(\log ^{+}|X|\right)^{\beta}\right\}<\infty$ for all $\beta<1$.

(viii) Let $\alpha>3 / 2$. Assume that $E|X|^{1 /(\alpha-1)}<\infty$ when $\alpha>2$. For $\alpha \leqslant 2$, assume that $E X=0$, that $E(|X| \log |X|)<\infty$ when $\alpha=2$, and that $E\left\{|X|^{1 /(\alpha-1)}\left(\log ^{+}|X|\right)^{\beta}\right\}$ $<\infty$ for some $\beta>(2-\alpha) /(\alpha-1)$ when $2>\alpha>3 / 2$. Then $P[S(\cdot)$ is continuously differentiable $]=1$. 
Note that the minimal assumption $E|X|^{1 / \alpha}<\infty$ in (1.3) for the a.s. convergence of $S(t)$ at every fixed $t$ is also sufficient for the a.s. uniform convergence of $S(\cdot)$ when $\alpha>1$, and that for $\alpha \leqslant 1$ the moment conditions in parts (iii), (iv), (v) of the above theorem are only slightly stronger than the minimal moment condition in (1.3). Moreover, for $\Phi_{n} \equiv 0$, the additional assumption $E X=0$ when $\alpha \leqslant 1$ is in fact necessary for the a.s. continuity of $S(\cdot)$, since $\Sigma_{1}^{\infty} n^{-\alpha} \sin n t$ is discontinuous at $t=0$ if $\alpha \leqslant 1$ (cf. [28, pp. 61, 70]) and therefore the a.s. continuity of $S(\cdot)$ is not preserved when $X_{n}$ is replaced by $X_{n}+\mu$ in (1.1) in this case. Parts (vi) and (vii) of the above theorem show that the moment conditions in parts (iii), (iv), (v) are in some sense minimal for the a.s. uniform convergence of $S(\cdot)$. These results are obtained as a special case of a general theorem on the a.s. unboundedness of $S(\cdot)$ proved in $\$ 3$. For independent (but not necessarily identically distributed) symmetric random variables $X_{n}$, classical results on the a.s. unboundedness of $S(\cdot)$ require the regularity condition $E X_{n}^{4}=O\left(\left(E X_{n}^{2}\right)^{2}\right)$ or $E X_{n}^{2}=O\left(E^{2}\left|X_{n}\right|\right)($ cf. [7, p. 135] and $[10$, p. 77]). We are able to remove this kind of regularity conditions in $\$ 3$ and thereby obtain Theorem 1 (vi), (vii).

One motivation for our investigation of the convergence and continuity of random Fourier series without the classical assumptions of finite variance and symmetry comes from the study of summability methods for i.i.d. random variables. Letting $X, X_{1}, \ldots$ be i.i.d., Kolmogorov's strong law of large numbers can be restated as follows: $E X=\mu$ iff the $(C, 1)$ limit of $X_{n}$ is $\mu$ a.s. This result has been extended in [14] to give the Cesàro $(C, \alpha)$ and Abel $(A)$ limits of $X_{n}$ : For $\alpha \geqslant 1$,

$$
\begin{aligned}
& E X=\mu \\
& \Leftrightarrow X_{n} \rightarrow \mu(C, \alpha) \text { a.s. } \quad\left(\text { i.e., } \lim _{n \rightarrow \infty} \sum_{1}^{n-1}\left(\begin{array}{c}
i+\alpha-1 \\
i
\end{array}\right) X_{n-i} /\left(\begin{array}{c}
n+\alpha \\
n
\end{array}\right)=\mu \text { a.s. }\right) \\
& \Leftrightarrow X_{n} \rightarrow \mu(A) \text { a.s. } \quad\left(\text { i.e., } \lim _{\lambda \rightarrow 1-}(1-\lambda) \sum_{1}^{\infty} \lambda^{i} X_{i}=\mu \text { a.s. }\right) .
\end{aligned}
$$

A summability method which involves series of the form (1.1) is Riemann's $R_{1}$ summability. Let $\left\{A_{n}\right\}$ be a sequence of real numbers. We say that $A_{n} \rightarrow A\left(R_{1}\right)$ if

$$
\lim _{t \rightarrow 0+}(2 / \pi) \sum_{1}^{\infty} n^{-1} A_{n} \sin n t=A
$$

(cf. [4]). By analogy with (1.6), it is natural to ask what kind of moment conditions on $X$ would be sufficient for $X_{n} \rightarrow \mu\left(R_{1}\right)$ a.s. Note that from the results of Kuttner [12], [13], $R_{1}$ summability implies $(C, \alpha)$ summability for every $\alpha>2$, and therefore in view of (1.6), $E X=\mu$ is a necessary condition for $X_{n} \rightarrow \mu\left(R_{1}\right)$ a.s. Viewing the summability method in (1.7) as some sort of "averaging operation" on the sequence $\left\{A_{n}\right\}$, it is natural to expect that the a.s. continuity of $\Sigma_{1}^{\infty} n^{-1} X_{n} \sin n t$ at $t=0$ or, of more fundamental interest, its a.s. continuity on $[0,2 \pi]$ may not involve much more than the necessary condition $E X=0$. Theorem 1(iv) shows that this is indeed the case, i.e., the slightly stronger assumption $E(|X| \log |X|)<\infty$ (in addition to $E X=0$ ) is sufficient for the a.s. continuity of $\sum n^{-1} X_{n} \sin n t$ on $[0,2 \pi]$ (although the condition $E X=0$ alone is not sufficient, as Theorem 1(vii) shows). 
The above point of view leads us to another interesting question concerning series of the form (1.1). First in connection with the rate of convergence in the $(C, 1)$ limit of $X_{n}$, the Hartman-Wintner law of the iterated logarithm says that

$$
\begin{aligned}
E X & =0 \text { and } E X^{2}=\sigma^{2} \\
& \Rightarrow \limsup _{n \rightarrow \infty}\left(\frac{n}{2 \log \log n}\right)^{1 / 2}\left|\frac{\sum_{1}^{n} X_{i}}{n}-\mu\right|=\sigma \quad \text { a.s., }
\end{aligned}
$$

and corresponding laws of the iterated logarithm for the $(C, \alpha)$ and the Abel limits of $X_{n}$ have been obtained in [14]. By analogy with these results, it is natural to expect that a similar law of the iterated logarithm would also hold for the $R_{1}$ limit of $X_{n}$. This indeed turns out to be the case. In fact, by specializing the results of $\$ 4$ to the particular case $a_{n}=n^{-\alpha}$ and $\Phi_{n}=0$, we obtain

Theorem 2. Let $\frac{1}{2}<\alpha<\frac{3}{2}$, and set $a_{n}=n^{-\alpha}$ and $\Phi_{n}=0$ in (1.1). If $E X=0$, $E X^{2}=\sigma^{2}$, and $E\left\{X^{2}\left(\log ^{+}|X|\right)^{p}\right\}<\infty$ for some $p>(6 \alpha+1) /(2 \alpha-1)$, then

$$
\underset{t \rightarrow 0}{\lim \sup } S(t) /\left\{2|t|^{2 \alpha-1}\left(\log \log |t|^{-1}\right)\right\}^{1 / 2}=A_{\alpha}^{1 / 2} \sigma \quad \text { a.s. }
$$

where $A_{\alpha}=\pi / 2$ if $\alpha=1$, and

$$
A_{\alpha}=-4^{\alpha-1} \Gamma(1-2 \alpha) \cos ((2 \alpha-1) \pi / 2) \text { if } \alpha \neq 1
$$

We note that the requirement $\alpha<3 / 2$ in Theorem 2 is natural since by Theorem 1 (viii), $S(\cdot)$ is a.s. continuously differentiable when $\alpha>3 / 2$. Obviously, replacing $X_{n}$ by $-X_{n}$ in Theorem 2 shows that the lim inf of the normalized $S(t)$ in the left-hand side of (1.8) is $-A_{\alpha}^{1 / 2} \sigma$ a.s. For the special case $\alpha=1$ in Theorem 2, we obtain the law of the iterated logarithm for the $R_{1}$ limit of the i.i.d. sequence $\left\{X_{n}\right\}$, i.e., if $E X=0, E X^{2}=\sigma^{2}$, and $E\left\{X^{2}\left(\log ^{+}|X|\right)^{p}\right\}<\infty$ for some $p>7$, then

$$
\limsup _{t \rightarrow 0}\left(\sum_{1}^{\infty} n^{-1} X_{n} \sin n t\right) /\left(2|t| \log \log |t|^{-1}\right)^{1 / 2}=(\pi / 2)^{1 / 2} \sigma \quad \text { a.s. }
$$

When $X$ is a standard normal random variable,

$$
W(t)=\pi^{-1 / 2} t X+(2 / \pi)^{1 / 2} \sum_{1}^{\infty} n^{-1} X_{n} \sin n t, \quad 0<t<\pi,
$$

is the standard Wiener process (cf. [1, p. 261]), and (1.10) reduces to the classical law of the iterated logarithm for Brownian motion. Thus Theorems 2 and 7 of $\$ 4$ are extensions of this important result to random Fourier series. Moreover, Theorem 2 provides an analogue (for random Fourier series) of Hunt's law of the iterated logarithm [5, Theorem 11] for the random Fourier-Wiener transform $\int_{0}^{\infty} u^{-\alpha}\left(e^{i u t}-1\right) d W(u)$.

2. Convergence and continuity. Throughout this section, almost sure (a.s.) convergence means convergence with probability 1 , while the phrase "almost everywhere" (a.e.) is with respect to Lebesgue measure on the real line. As pointed out in $\S 1$, if $X$ is symmetric and has a finite variance $\sigma^{2} \neq 0$, then (1.2) is necessary and sufficient for the a.s. uniform convergence of $S(\cdot)$. In general, without assuming $X$ to be symmetric or to have a finite variance, (1.2) is still necessary for $S(\cdot)$ to 
converge everywhere a.s. if $X$ is nondegenerate, as is shown by the following

LEMMA 1. If $X$ is nondegenerate, then

$$
P[S(t) \text { converges for every } t]=1 \Rightarrow\left\{a_{n}\right\} \text { satisfies (1.2). }
$$

Proof. Let $X_{1}^{*}, X_{2}^{*}, \ldots$ be i.i.d. and independent of the sequence $\left\{\left(X_{n}, \Phi_{n}\right)\right\}$ such that $X_{n}^{*}$ and $X_{n}$ have the same distribution. Let

$$
S^{*}(t)=\sum a_{n}\left(X_{n}-X_{n}^{*}\right) \sin \left(n t+\Phi_{n}\right)
$$

If $S(t)$ converges everywhere a.s., then so does $S^{*}(t)$. Since $X_{n}-X_{n}^{*}$ is symmetric and is independent of $\Phi_{n}$, this in turn implies by Billard's theorem [10, p. 49] that $P\left[S^{*}(t)\right.$ converges uniformly in $\left.t\right]=1$. Applying Corollary 3.2 and Theorem 4.1 of [18] together with Corollary 2.8 of [20], it follows from the a.s. uniform convergence of $S^{*}(t)$ that $\left\{a_{n}\right\}$ satisfies (1.2), thus completing the proof.

Without the assumptions of symmetry and finite variance on $X$, the condition (1.2) on $\left\{a_{n}\right\}$ is, however, not sufficient for the a.s. uniform convergence of $S(\cdot)$. To give an example showing this, let $a_{n}=n^{-\alpha}\left(\alpha>\frac{1}{2}\right), E|X|^{1 / \alpha}=\infty$ and $\Phi_{n}=0$. Then (1.2) is satisfied (see Remark 2.7 of [20]), but $S(t)$ does not even converge a.s. at $t=\frac{1}{2} \pi$, as is shown in the following

Proof of (1.3). Assume that $S\left(\frac{1}{2} \pi\right)=\Sigma_{1}^{\infty} n^{-\alpha} X_{n} \sin (n \pi / 2)$ converges a.s. Then $\lim _{m \rightarrow \infty}(2 m+1)^{-\alpha} X_{2 m+1}=0$ a.s. and hence $E|X|^{1 / \alpha}<\infty$ by the Borel-Cantelli lemma. To complete the proof of (1.3), we now assume conversely that $E|X|^{1 / \alpha}<$ $\infty$. First consider the case $\frac{1}{2}<\alpha<1$ and note that our assumption implies that $E|X|<\infty$. Since $\sum_{1}^{\infty} n^{-\alpha} \sin n t$ converges for every $t$ (cf. [28, pp. 61, 70]), we can assume that $E X=0$. With this assumption, define

$$
X_{n}^{\prime}=X_{n} I_{\left[\left|X_{n}\right|<n^{\alpha}\right]}-E X I_{\left[|X|<n^{\alpha}\right]} \text {. }
$$

Since $E|X|^{1 / \alpha}<\infty$, it follows that

$$
\sum_{1}^{\infty} E\left(n^{-\alpha} X_{n}^{\prime}\right)^{2}<E\left\{X^{2}\left(\sum_{|X|<n^{\alpha}} n^{-2 \alpha}\right)\right\}<\infty,
$$

and therefore $\Sigma n^{-\alpha} X_{n}^{\prime} \sin n t$ converges a.s. We note that since $E X=0$,

$$
\begin{aligned}
\sum_{1}^{N} n^{-\alpha}(\sin n t) E X I_{\left[|X|<n^{\alpha}\right]} & =-\sum_{1}^{N} n^{-\alpha}(\sin n t) E X I_{\left[|X|>n^{\alpha}\right]} \\
& =-E\left\{X \sum_{n<\min \left(N,|X|^{1 / \alpha}\right)} n^{-\alpha} \sin n t\right\} .
\end{aligned}
$$

Since $\Sigma n^{-\alpha} \sin n t$ converges and $E|X|<\infty,(2.1)$ implies that

$$
\sum_{1}^{N} n^{-\alpha}(\sin n t) E X I_{\left[|X|<n^{\alpha}\right]} \text { converges as } N \rightarrow \infty \text {. }
$$

Noting that $P\left[\left|X_{n}\right|<n^{\alpha}\right.$ for all large $\left.n\right]=1$ by the Borel-Cantelli lemma, the a.s. convergence of $\Sigma_{1}^{\infty} n^{-\alpha} X_{n} \sin n t$ follows in the case $\frac{1}{2}<\alpha<1$. The case $\alpha>1$ is now treated in the following 
Proof of Theorem 1(ii). Since $\alpha>1, \Sigma_{1}^{\infty} n^{-\alpha}<\infty$ and an application of the three-series theorem shows that $\Sigma\left|n^{-\alpha} X_{n}\right|<\infty$ a.s. if $E|X|^{1 / \alpha}<\infty$. Hence the desired conclusion follows.

Suppose that $X$ is symmetric and has a finite variance $\sigma^{2}>0$. Then it follows from the Paley-Zygmund theorem [10, p. 45] that for any sequence of constants $a_{n}$,

$$
P[S(t) \text { converges for almost every } t]=1 \Leftrightarrow \sum_{1}^{\infty} a_{n}^{2}<\infty \text {. }
$$

In view of (2.3) we shall assume that $\left\{a_{n}\right\} \in l^{2}$ in connection with questions on the a.s. convergence of $S(t)$. Given $\left\{a_{n}\right\} \in l^{2}$, the following theorem gives conditions on the tail distribution of $X$ which guarantee the a.s. pointwise or uniform convergence of the random Fourier series $S(t)$ as defined in (1.1).

THEOREM 3. (i) Let $\left\{a_{n}\right\} \in l^{2}$. If

$$
\sum_{1}^{\infty} E\left(\min \left\{\left|a_{n} X\right|^{2}, 1\right\}\right)<\infty
$$

then

$$
P[S(t) \text { converges for almost every } t]=1 \text {. }
$$

Conversely, if

$$
\text { either } X \text { is symmetric or } e^{i \Phi_{n}} \text { is symmetric for all } n \text {, }
$$

then (2.5) implies (2.4).

(ii) Suppose that there exist nonnegative constants $b_{n}$ such that

$$
\begin{gathered}
\sum_{1}^{\infty} a_{n}^{2} E\left(X^{2} I_{\left[|X|<b_{n}\right]}\right)<\infty, \text { and for some } 0<\delta<1, \\
\int_{0}^{\delta}\left[M\left(\left\{\sum_{n=1}^{\infty} a_{n}^{2} E\left(X^{2} I_{\left[|X|<b_{n}\right]}\right) \sin ^{2} \frac{n t}{2}\right\}^{1 / 2}\right) /\left\{t|\log t|^{1 / 2}\right\}\right] d t<\infty, \\
\sum_{n=1}^{\infty} E\left\{\min \left(\left|a_{n} X\right|, 1\right) I_{\left[|X|>b_{n}\right]}\right\}<\infty
\end{gathered}
$$

and

$$
\sum_{n=1}^{\infty}\left|a_{n} E\left(X I_{\left[|X|<b_{n}\right]}\right)\right|\left|E e^{i \Phi_{n}}\right|<\infty
$$

then

$$
P[S(t) \text { converges uniformly in } t]=1 \text {. }
$$

REMARKS. (i) If the sequence $\left\{a_{n}^{2} E\left(X^{2} I_{\left[|X|<b_{n}\right.}\right)\right\}$ is nonincreasing, then (2.7) is equivalent to

$$
\sum_{n=2}^{\infty}\left[\left\{\sum_{k=n}^{\infty} a_{k}^{2} E\left(X^{2} I_{\left[|X|<b_{k}\right]}\right)\right\}^{1 / 2} /\left\{n(\log n)^{1 / 2}\right\}\right]<\infty
$$

(see Remark 2.7 of [20]). In general, without the monotonicity assumption on $\left\{a_{n}^{2} E\left(X^{2} I_{\left[|X|<b_{n}\right]}\right)\right\}$, Marcus [17, Theorem 1] has shown that $\left(2.7^{*}\right) \Rightarrow(2.7)$. 
(ii) Under the symmetry assumption (2.6), (2.9) holds and (2.10) is equivalent by Billard's theorem [10, p. 49] to

$$
P[S(t) \text { converges for every } t]=1 \text {. }
$$

(iii) Obviously (2.7) and (2.8) imply (2.4). On the other hand, if (2.4) is strengthened into

$$
\sum_{n=2}^{\infty}\left[\left\{\sum_{k=n}^{\infty} E \min \left(\left|a_{k} X\right|^{2}, 1\right)\right\}^{1 / 2} /\left\{n(\log n)^{1 / 2}\right\}\right]<\infty
$$

then by choosing

$$
b_{n}=\left|a_{n}\right|^{-1} \text { if } a_{n} \neq 0 \quad\left(b_{n} \text { arbitrary if } a_{n}=0\right),
$$

it is easy to see that $\left(2.7^{*}\right)$ and (2.8) both hold.

As an immediate corollary of Theorem 3, we obtain the following

CoROllary 1. Let $\left\{a_{n}\right\} \in l^{2}$ such that (1.2) holds. If $E X=0$ and $E X^{2}<\infty$, then (2.10) holds.

Proof. The conditions (2.7)-(2.9) are satisfied with $b_{n}$ defined by (2.13), noting that

$$
\sum_{n=1}^{\infty}\left|a_{n}\right| E\left(|X| I_{\left[\left|a_{n} X\right|>1\right]}\right)<\sum_{n=1}^{\infty} a_{n}^{2} E X^{2}<\infty
$$

As another application of Theorem 3(ii), we obtain the following result which implies Theorem 1(iii), (iv) and (viii).

COROLlaRY 2. Suppose that $\left\{a_{n}\right\} \in l^{2}$ such that for some $\gamma>0$,

$$
\sum_{1}^{\infty} a_{n}^{2}(\log n)^{1+\gamma}<\infty
$$

For $x \geqslant 0$, define

$$
\begin{aligned}
& \psi(x)=\min \left\{n \geqslant 1: \sum_{k=1}^{n}\left|a_{k}\right| \geqslant x \sum_{k=n}^{\infty} a_{k}^{2}(\log k)^{1+\gamma}\right\}, \\
& \varphi(x)=\sum_{k=1}^{\psi(x)}\left|a_{k}\right| .
\end{aligned}
$$

Then $\varphi(x)=o(x)$ as $x \rightarrow \infty$. If $E\{|X| \varphi(|X|)\}<\infty$ and $E X=0$, then (2.10) holds.

Proof. Without loss of generality we can assume that $\sum_{k=n}^{\infty} a_{k}^{2}>0$ for all $n$. Then $\psi(x) \uparrow \infty$. Define $b_{k}=\inf \{x \geqslant 0: \psi(x) \geqslant k\}$. Then

$$
\sum_{k=1}^{\infty} E\left\{\left|a_{k} X\right| I_{\left[|X|>b_{k}\right]}\right\}=E\left\{|X|\left(\sum_{k=1}^{\psi(|X|)}\left|a_{k}\right|\right)\right\}=E\{|X| \varphi(|X|)\}<\infty
$$


and therefore (2.8) and (2.9) both hold, noting that $E X=0$. Moreover,

$$
\begin{aligned}
\sum_{k=1}^{\infty} E\left\{a_{k}^{2} X^{2}(\log k)^{1+\gamma} I_{\left[|X|<b_{k}\right]}\right\} & \leqslant E\left\{X^{2}\left(\sum_{k=\psi(|X|)}^{\infty} a_{k}^{2}(\log k)^{1+\gamma}\right)\right\} \\
& \leqslant E\left\{|X|\left(\sum_{k=1}^{\psi(|X|)}\left|a_{k}\right|\right)\right\}, \text { by (2.15), } \\
& =E\{|X| \varphi(|X|)\}<\infty .
\end{aligned}
$$

It is easy to see that $(2.18)$ implies $\left(2.7^{*}\right)$. Therefore by Theorem $3(\mathrm{ii})$, the desired conclusion follows.

We note that for the case $a_{n}=n^{-\alpha}\left(\alpha>\frac{1}{2}\right)$, the condition (2.4) holds iff $E|X|^{1 / \alpha}<\infty$. Hence the first part of Theorem 1(i) is a special case of Theorem 3(i). Moreover, this choice of $a_{n}$ satisfies the condition (2.14) for all $\gamma>0$, and as $x \rightarrow \infty$,

$$
\begin{aligned}
\varphi(x) & \sim C_{\alpha} x^{(1 / \alpha)-1}(\log x)^{(1+\gamma)(1-\alpha) / \alpha} \quad\left(\frac{1}{2}<\alpha<1\right), \\
& \sim C_{\alpha} \log x \quad(\alpha=1),
\end{aligned}
$$

where $C_{\alpha}$ is a positive constant depending on $\alpha$ and $\varphi$ is as defined in (2.16). Hence Theorem 1(iii), (iv) follow immediately from Corollary 2. Theorem 1(ii)-(iv) can in turn be used to check that $P\left[\sum_{1}^{\infty} n^{1-\alpha} X_{n} \cos \left(n t+\Phi_{n}\right)\right.$ converges uniformly in $t]=1$ in Theorem 1(viii) and therefore the desired a.s. differentiability of $\sum_{1}^{\infty} n^{-\alpha} X_{n} \sin \left(n t+\Phi_{n}\right)$ follows.

As another application of Theorem 3(ii), we obtain the following

Proof of Theorem 1(v). Assume that $E\{|X| \log \log (|X|+e)\}<\infty$. Take $\beta>1$ and let $b_{k}=k /(\log k)^{\beta}$ for $k>3$. Since $a_{k}=k^{-1}$,

$$
\begin{aligned}
\sum_{k=3}^{\infty} E\{ & \left.\min \left(\left|a_{k} X\right|, 1\right) I_{\left[|X|>b_{k}\right]}\right\} \\
& \leqslant \sum_{1}^{\infty} P\left[\left|k^{-1} X\right|>1\right]+E\left\{|X|\left(\sum_{b_{k}<|X|<k} k^{-1}\right)\right\} .
\end{aligned}
$$

Noting that for all large $|x|$

$$
\sum_{b_{k}<|x|<k} k^{-1}<\sum_{|x|<k<2|x|(\log |x|)^{\beta}} k^{-1} \sim \beta \log \log |x|
$$

we obtain (2.8). For all large $n$,

$$
\begin{aligned}
\sum_{k=n}^{\infty} k^{-2} E\left(X^{2} I_{\left[|X|<b_{k}\right]}\right)< & \left(\sum_{k=n}^{\infty} k^{-2}\right) E\left(X^{2} I_{\left[|X|<b_{n}\right]}\right) \\
& +E\left\{X^{2}\left(\sum_{|X|<b_{k}} k^{-2}\right) I_{\left[|X|>b_{n}\right]}\right\} \\
= & O\left((\log n)^{-\beta}\right) .
\end{aligned}
$$

Since $\beta>1,\left(2.7^{*}\right)$ follows. Since the symmetry condition (2.6) is assumed, (2.9) holds. Therefore the desired conclusion follows from Theorem 3(ii). 
The following simple example shows that it is not enough to replace the symmetry assumption (2.6) in Theorem $1(v)$ by the assumption $E X=0$. In this connection, it also illustrates the significance of the assumption (2.9) in Theorem 3(ii).

EXAMPLE. Let $a_{n}=n^{-1}$ and $\Phi_{n}=\theta$, where $0<\theta<\pi$. Suppose that the random variable $X$ has the following distribution:

$$
P[X=-1]=c, \quad P[X>t]=d \int_{t}^{\infty} f(x) d x \quad \text { for } t>2,
$$

where $f(x)=1 /\left\{x^{2}(\log x)^{2}\right\}$ for $x \geqslant 2$, and $c, d$ are positive numbers defined by the equations

$$
c+d \int_{2}^{\infty} f(x) d x=1, \quad c=d \int_{2}^{\infty} x f(x) d x .
$$

Then $E X=0$ and

$$
\begin{aligned}
E\left\{|X|\left(\log ^{+}|X|\right)^{\beta}\right\} & <\infty \quad \text { if } \beta<1, \\
& =\infty \quad \text { if } \beta>1 .
\end{aligned}
$$

Define $b_{k}=k /\{(\log k)(\log \log k)\}$. Then by the argument used in the proof of Theorem 1(v), it can be shown that the assumptions $\left(2.7^{*}\right)$ and $(2.8)$ are satisfied. However, noting that $S(t)=\sum n^{-1} X_{n} \sin (n t+\theta)$ and that $\sin \theta \neq 0$, we obtain that

$$
P[S(t) \text { converges at } t=0]=P\left[\sum_{1}^{\infty} n^{-1} X_{n} \text { converges }\right]=0
$$

by the three-series theorem, since

$$
\sum_{1}^{\infty} n^{-1} E X_{n} I_{\left[\left|n^{-1} X_{n}\right|<1\right]}=-\sum_{1}^{\infty} n^{-1} E X I_{[X>n]}=-\infty .
$$

In view of (2.20), the above example also proves the zero-mean part of Theorem 1(vii), and we have already proved Theorem l(i)-(v), (viii) by making use of Theorem 3 and its corollaries. We now give the proof of Theorem 3, which we preface by the following result of Marcus [19, Theorem 1.3]:

LEMMA 2. Let $\left\{\left(Z_{n}, \Phi_{n}\right)\right\}$ be a sequence of independent bivariate random vectors such that $E\left(Z_{n} e^{i \Phi_{n}}\right)=0$ for all $n$ and $\Sigma_{1}^{\infty} E Z_{n}^{2}<\infty$. If for some $0<\delta<1$

$$
\int_{0}^{\delta}\left[M\left(\left\{\sum_{n=1}^{\infty}\left(E Z_{n}^{2}\right) \sin ^{2}\left(\frac{n t}{2}\right)\right\}^{1 / 2}\right) /\left\{t|\log t|^{1 / 2}\right\}\right] d t<\infty,
$$

then $P\left[\Sigma_{1}^{\infty} Z_{n} \sin \left(n t+\Phi_{n}\right)\right.$ converges uniformly in $\left.t\right]=1$.

Proof of Theorem 3. Under the symmetry assumption (2.6), the equivalence between (2.4) and (2.5) follows from the Paley-Zygmund theorem, as has been shown in $[10$, p. 45]. We now use a different argument to prove $(2.4) \Rightarrow(2.5)$ without the symmetry assumption (2.6). Note that (2.4) is equivalent to

$$
\sum_{1}^{\infty}\left(a_{n} X_{n}\right)^{2}<\infty \quad \text { a.s. }
$$


(cf. [10, p. 27]). Hence (2.4) implies that with probability $1,\left\{a_{n} X_{n} \cos \Phi_{n}\right\} \in l^{2}$ and $\left\{a_{n} X_{n} \sin \Phi_{n}\right\} \in l^{2}$. Therefore by Carleson's theorem [2], (2.5) holds.

To prove (ii), let $X_{n}^{\prime}=X_{n} I_{\left[\left|X_{n}\right|<b_{n}\right]}, X_{n}^{\prime \prime}=X_{n} I_{\left[\left|X_{n}\right|>b_{n}\right]}$. By (2.8), $\Sigma_{1}^{\infty}\left|a_{n} X_{n}^{\prime \prime}\right|<\infty$ a.s. (cf. [10, p. 27]). By (2.9) and the independence of $\left\{X_{n}\right\}$ and $\left\{\Phi_{n}\right\}$,

$$
\sum_{1}^{\infty}\left|a_{n} E\left(X_{n}^{\prime} \cos \Phi_{n}\right)\right|+\sum_{1}^{\infty}\left|a_{n} E\left(X_{n}^{\prime} \sin \Phi_{n}\right)\right|<\infty \text {. }
$$

Therefore it suffices to show that

$$
\begin{gathered}
\sum a_{n}\left(X_{n}^{\prime} \cos \Phi_{n}-E X_{n}^{\prime} \cos \Phi_{n}\right) \sin n t \text { and } \\
\sum a_{n}\left(X_{n}^{\prime} \sin \Phi_{n}-E X_{n}^{\prime} \sin \Phi_{n}\right) \cos n t \quad \text { converge uniformly a.s. }
\end{gathered}
$$

Since $E\left(X_{n}^{\prime}\right)^{2} \geqslant \max \left\{\operatorname{Var}\left(X_{n}^{\prime} \sin \Phi_{n}\right), \operatorname{Var}\left(X_{n}^{\prime} \cos \Phi_{n}\right)\right\}$, the desired conclusion (2.22) follows from (2.7) and Lemma 2.

3. Conditions for unboundedness. Consider the random Fourier series

$$
\sum_{1}^{\infty} Z_{n} \sin \left(n t+\Phi_{n}\right)
$$

where $\left\{\left(Z_{n}, \Phi_{n}\right)\right\}$ is a sequence of independent bivariate random vectors. We say that (3.1) is unbounded a.s. if

$$
P\left[\sup _{N} \max _{t}\left|\sum_{1}^{N} Z_{n} \sin \left(n t+\Phi_{n}\right)\right|=\infty\right]=1 .
$$

In the case where $Z_{n} e^{i \Phi_{n}}$ are symmetric and

$$
E Z_{n}^{4} \leqslant C\left(E Z_{n}^{2}\right)^{2}<\infty \text { for some } C>0 \text { and all } n,
$$

Kahane [10] showed that a sufficient condition for (3.2) to hold is

$$
\sum_{k=1}^{\infty}\left\{\sum_{n=2^{k}}^{2^{k+1}-1} E Z_{n}^{2}\right\}^{1 / 2}=\infty
$$

Recently Jain and Marcus [7] replaced the assumption (3.3) by the assumption

$$
E Z_{n}^{2}<C\left(E\left|Z_{n}\right|\right)^{2}<\infty \text { for some } C>0 \text { and all } n,
$$

but they needed an additional assumption that $E Z_{n}^{2} \downarrow$ (i.e., $E Z_{n}^{2}$ be nonincreasing). However, in our subsequent applications where we show that the moment conditions in Theorem 1 are in some sense nearly minimal, the regularity conditions (3.3) and (3.5) fail to hold. In the following theorem we remove these regularity conditions and use the condition (3.4) in a slightly stronger form.

THEOREM 4. Let $\left\{\left(Z_{n}, \Phi_{n}\right)\right\}$ be a sequence of independent bivariate random vectors such that

$$
\begin{aligned}
& E Z_{n}=0 \text { for all } n, \\
& \sum_{1}^{\infty} E Z_{n}^{2}<\infty, \text { and } \\
& \sum_{k=1}^{\infty} E\left|\sum_{n=2^{k}}^{2^{k+1}-1} Z_{n}\right|=\infty
\end{aligned}
$$


(i) If $Z_{n} e^{i \Phi_{n}}$ is symmetric for every $n$, then (3.2) holds, and consequently

$$
P\left[\sum_{1}^{\infty} Z_{n} \sin \left(n t+\Phi_{n}\right) \text { converges at every } t\right]=0 \text {, and }
$$

$P\left[\sum_{1}^{\infty} Z_{n} \sin \left(n t+\Phi_{n}\right)\right.$ is the Fourier-Lebesgue series of a bounded function $]=0$.

(ii) If $Z_{n}$ and $\Phi_{n}$ are independent for every $n$, then (3.2), (3.9), and (3.10) still hold (without any assumptions of symmetry).

Remarks. (i) Since $E Z_{n}=0$, the Marcinkiewicz-Zygmund inequalities [16] imply that there exist absolute constants $A, B(>0)$ such that

$$
A E\left\{\left(\sum_{n=2^{k}}^{2^{k+1}-1} Z_{n}^{2}\right)^{1 / 2}\right\}<E\left|\sum_{n=2^{k}}^{2^{k+1}-1} Z_{n}\right|<B E\left\{\left(\sum_{n=2^{k}}^{2^{k+1}-1} Z_{n}^{2}\right)^{1 / 2}\right\}
$$

Hence the condition (3.8) is equivalent to

$$
\sum_{k=1}^{\infty} E\left\{\left(\sum_{n=2^{k}}^{2^{k+1}-1} Z_{n}^{2}\right)^{1 / 2}\right\}=\infty
$$

Since $E\left\{\left(\sum_{n=l}^{m} Z_{n}^{2}\right)^{1 / 2}\right\} \leqslant\left\{\sum_{n=l}^{m} E Z_{n}^{2}\right\}^{1 / 2}$, the condition (3.12) implies the condition (3.4).

(ii) Suppose that $E Z_{n}=0$ and that (3.3) holds. Then by the Paley-Zygmund inequalities [10, p. 24],

$$
P\left[\left|\sum_{n=2^{k}}^{2^{k+1}-1} Z_{n}\right|>\frac{1}{2}\left(\sum_{n=2^{k}}^{2^{k+1}-1} E Z_{n}^{2}\right)^{1 / 2}\right]>\left(\frac{3}{4}\right)^{2} \min \left(\frac{1}{3}, C^{-1}\right),
$$

and therefore

$$
E\left|\sum_{n=2^{k}}^{2^{k+1}-1} Z_{n}\right| \geqslant\left(\frac{3}{4}\right)^{2} \min \left\{1 / 6,(2 C)^{-1}\right\}\left(\sum_{n=2^{k}}^{2^{k+1}-1} E Z_{n}^{2}\right)^{1 / 2} .
$$

Hence (3.8) is equivalent to the classical condition (3.4) under the assumption (3.3) of Kahane.

(iii) Suppose that $E Z_{n}=0, E Z_{n}^{2} \downarrow$, and (3.5) holds. Then as shown in [7, pp. 136-137], the condition (3.4) implies that

$$
\sum_{k=1}^{\infty}\left|\sum_{n=2^{k}}^{2^{k+1}-1} Z_{n}\right|=\infty \quad \text { a.s. }
$$

and therefore a fortiori (3.8) holds. Hence (3.8) is equivalent to the condition (3.4) under the assumptions (3.5) and $E Z_{n}^{2} \downarrow$ of Jain and Marcus.

We preface the proof of Theorem 4 by the following three lemmas.

LEMMA 3. Let $W$ be a symmetric random variable independent of the random variables $U$ and $V$. If $|U|$ is stochastically larger than $|V|$ (i.e., $P[|U|>x]>P[|V|$ $>x]$ for all $x$, then $E|W+U|>E|W+V|$. 
Proof. Let $F(w)$ be the distribution function of $W$ and let $g(x)=E|W+x|$. Since $W$ is symmetric, $g$ is an even function. Moreover, for $x>0$,

$$
\begin{aligned}
g(x) & =E|W+x|=\int_{0}^{\infty}(|w+x|+|-w+x|) d F(w) \\
& =2 \int_{0}^{\infty} \max (w, x) d F(w) .
\end{aligned}
$$

Therefore $g(|x|)$ is nondecreasing in $|x|$. Hence

$$
E|W+U|=E g(\mid U)>E g(|\eta|)=E \mid W+\eta \text {. }
$$

LEMMA 4. Suppose that the random variables $U_{1}, \ldots, U_{n}$ are independent and symmetric, and that the same is true for the random variables $V_{1}, \ldots, V_{n}$. If $\left|U_{i}\right|$ is stochastically larger than $\left|V_{i}\right|$ for $i=1, \ldots, n$, then

$$
E\left|U_{1}+\cdots+U_{n}\right|>E\left|V_{1}+\cdots+V_{n}\right| \text {. }
$$

Proof. By Lemma 3,

$$
E\left|V_{1}+\cdots+V_{n}\right|<E\left|\left(V_{1}+\cdots+V_{n-1}\right)+U_{n}\right|<\cdots<E\left|U_{1}+\cdots+U_{n}\right| \text {. }
$$

LEMMA 5. Let $U_{1}, \ldots, U_{n}, I_{1}, \ldots, I_{n}$ be independent random variables such that $P\left[I_{j}=1\right]=P\left[I_{j}=0\right]=\frac{1}{2}$ for $j=1, \ldots, n$. Then $E\left|\sum_{j=1}^{n} U_{j} I_{j}\right| \geqslant \frac{1}{2} E\left|\sum_{j=1}^{n} U_{j}\right|$.

Proof. Note that $E\left|\Sigma_{1}^{n} U_{j}\right|<E\left|\Sigma_{1}^{n} I_{j} U_{j}\right|+E\left|\Sigma_{1}^{n}\left(1-I_{j}\right) U_{j}\right|$ and that $I_{j} U_{j}$ and $\left(1-I_{j}\right) U_{j}$ have the same distribution.

Proof of TheOREM 4(i). First consider the particular case where $\Phi_{n}$ is uniformly distributed in $[0,2 \pi]$ and $Z_{n}$ is symmetric and independent of $\Phi_{n}(n=1,2, \ldots)$. Define

$$
\begin{gathered}
f(x)=(1-|x|)^{+}, \quad \theta(x)=\prod_{n=0}^{\infty} f\left(2^{-n} x\right), \\
S_{j}(t)=\sum_{n=1}^{2^{j}-1} Z_{n} \theta\left(2^{-(j+2)} n\right) \sin \left(n t+\Phi_{n}\right), \\
S_{j}^{*}(t)=\sum_{n=1}^{2^{j}-1} Z_{n} \theta\left(2^{-j} n\right) \sin \left(n t+\Phi_{n}\right), \quad H_{j}^{*}=\max _{t} S_{j}^{*}(t), \\
P_{j}(t)=\sum_{n=2^{j}}^{2^{j+2}-1} Z_{n} \theta\left(2^{-(j+2)} n\right) \sin \left(n t+\Phi_{n}\right), P_{j}^{+}(t)=\left(P_{j}(t)\right)^{+}, \\
t_{j}=\inf _{t}\left\{0: S_{j}(t)>H_{j}^{*}\right\} \quad(\inf \varnothing=\infty) .
\end{gathered}
$$

Then by an argument similar to that in [10, pp. 74-75], $S_{j+2}^{*}(t)=S_{j}(t)+P_{j}(t)$, $\max _{t} S_{j}(t) \geqslant H_{j}^{*}$ and therefore $t_{j}<\infty$; moreover, $H_{j+2}^{*}-H_{j}^{*}>S_{j+2}^{*}\left(t_{j}\right)-S_{j}\left(t_{j}\right)=$ $P_{j}\left(t_{j}\right)$. Since $H_{j}^{*} \uparrow$ (cf. [10, p. 74]), this implies that $H_{j+2}^{*}-H_{j}^{*}>P_{j}^{+}\left(t_{j}\right)$, and therefore

$$
2 H_{k+2}^{*}>H_{k+2}^{*}+H_{k+1}^{*}>H_{1}^{*}+H_{2}^{*}+\sum_{1}^{k} P_{j}{ }^{+}\left(t_{j}\right) .
$$


We now show that as $k \rightarrow \infty$,

$$
\begin{aligned}
E\left\{\sum_{1}^{k} P_{j}^{+}\left(t_{j}\right)\right\} & \rightarrow \infty, \text { and } \\
\operatorname{Var}\left\{\sum_{1}^{k} P_{j}^{+}\left(t_{j}\right)\right\} & =O(1) .
\end{aligned}
$$

Let $\mathcal{F}_{j}$ denote the $\sigma$-field generated by $\left\{\left(Z_{n}, \Phi_{n}\right): n<2^{j}-1\right\}$. Then the random function $P_{j}(\cdot)$ is independent of $\mathscr{F}_{j}$ and $t_{j}$ is $\mathscr{F}_{j}$-measurable. Since $\Phi_{n}$ is uniform in $[0,2 \pi]$ and is independent of $Z_{n}$, it follows that $P_{j}(t)$ has the same distribution for every fixed $t$, and therefore $P_{j}\left(t_{j}\right)$ has the same distribution as $P_{j}(0)$. Moreover, since $Z_{n}$ is symmetric, we have

$$
E P_{j}^{+}\left(t_{j}\right)=E P_{j}^{+}(0)=E P_{j}^{-}(0)=\frac{1}{2} E\left|P_{j}(0)\right| \text {. }
$$

To prove (3.15), let $I_{n}=I_{\left[\left|\sin \Phi_{n}\right|>2^{-1 / 2}\right]}$. Then $P\left[I_{n}=1\right]=P\left[I_{n}=0\right]=\frac{1}{2}$ and $\left|Z_{n} \sin \Phi_{n}\right| \geqslant 2^{-1 / 2}\left|Z_{n} I_{n}\right|$. Moreover $Z_{n} I_{n}$ is symmetric and $Z_{n}$ is independent of $I_{n}$. Therefore by Lemma 4,

$$
\begin{aligned}
E\left|P_{j}(0)\right| & \geqslant 2^{-1 / 2} E\left|\sum_{n=2^{j}}^{2^{j+2}-1} Z_{n} I_{n} \theta\left(2^{-j+2)} n\right)\right| \\
& >2^{-1 / 2} \theta\left(\frac{1}{2}\right) E\left|\sum_{n=2^{j}}^{2^{j+1}-1} Z_{n} I_{n}\right| \\
& >\frac{1}{4} \theta\left(\frac{1}{2}\right) E\left|\sum_{n=2^{j}}^{2^{j+1}-1} Z_{n}\right|, \text { by Lemma } 5 .
\end{aligned}
$$

From (3.8), (3.17), and (3.18), (3.15) follows.

To prove (3.16), we first note that for $j>i+2$,

$$
\begin{aligned}
E\left(P_{j}^{+}\left(t_{j}\right) P_{i}^{+}\left(t_{i}\right)\right) & =E\left\{P_{i}^{+}\left(t_{i}\right) E\left[P_{j}^{+}\left(t_{j}\right) \mid \mathscr{F}_{j}\right]\right\} \\
& =\left(E P_{i}^{+}\left(t_{i}\right)\right)\left(E P_{j}^{+}(0)\right)=\left(E P_{i}^{+}\left(t_{i}\right)\right)\left(E P_{j}^{+}\left(t_{j}\right)\right),
\end{aligned}
$$

and therefore

$$
P_{j}^{+}\left(t_{j}\right) \text { and } P_{i}^{+}\left(t_{i}\right) \text { are uncorrelated if }|i-j|>2 \text {. }
$$

Moreover,

$$
\operatorname{Var} P_{j}^{+}\left(t_{j}\right)<E\left|P_{j}\left(t_{j}\right)\right|^{2}=E\left|P_{j}(0)\right|^{2}<\sum_{n=2^{j}}^{2^{j+2}-1} E Z_{n}^{2} .
$$

Using the inequality $\operatorname{Var}(U+V)<2(\operatorname{Var} U+\operatorname{Var} V)$, we obtain that

$$
\begin{aligned}
\operatorname{Var}\left\{\sum_{1}^{k} P_{j}^{+}\left(t_{j}\right)\right\} & <2\left[\operatorname{Var}\left\{\sum_{j \text { odd } j<k} P_{j}^{+}\left(t_{j}\right)\right\}+\operatorname{Var}\left\{\sum_{j \text { even } j<k} P_{j}^{+}\left(t_{j}\right)\right\}\right] \\
& <4 \sum_{n=1}^{2^{k+2}} E Z_{n}^{2}, \quad \text { by (3.19) and (3.20). }
\end{aligned}
$$

From (3.7) and (3.21), (3.16) follows. 
From (3.15) and (3.16), it follows that

$$
\left\{E\left(\sum_{1}^{k} P_{j}^{+}\left(t_{j}\right)\right)\right\}^{2} / E\left\{\sum_{1}^{k} P_{j}^{+}\left(t_{j}\right)\right\}^{2} \rightarrow 1 \quad \text { as } k \rightarrow \infty .
$$

Using the inequality $P[Y \geqslant \lambda E Y] \geqslant(1-\lambda)^{2}(E Y)^{2} /\left(E Y^{2}\right)$ for $0<\lambda<1$ and $Y \in L^{2}$ such that $Y \geqslant 0$ a.s. (cf. [10, p. 6]), we obtain from (3.22) that as $k \rightarrow \infty$,

$$
\begin{aligned}
P\left[\sum_{1}^{\infty} P_{j}^{+}\left(t_{j}\right)\right. & \left.\geqslant \lambda E\left\{\sum_{1}^{k} P_{j}^{+}\left(t_{j}\right)\right\}\right] \\
& \geqslant P\left[\sum_{1}^{k} P_{j}^{+}\left(t_{j}\right) \geqslant \lambda E\left\{\sum_{1}^{k} P_{j}^{+}\left(t_{j}\right)\right\}\right] \geqslant(1-\lambda)^{2}+o(1)
\end{aligned}
$$

for every fixed $0<\lambda<1$. From (3.15) and (3.23), it then follows that $P\left[\Sigma_{1}^{\infty} P_{j}^{+}\left(t_{j}\right)\right.$ $=\infty]=1$. In view of (3.14), this in turn implies that $P\left[\lim _{j \rightarrow \infty} H_{j}^{*}=\infty\right]=1$. Since $H_{j}^{*} \leqslant \max _{t} \sum_{n<2^{j}} Z_{n} \sin \left(n t+\Phi_{n}\right.$ ) (cf. [10, p. 74]), we have proved (3.2), and therefore by Billard's theorem [10, p. 49], (3.9) and (3.10) also hold.

Now returning to general case where the $\left(Z_{n}, \Phi_{n}\right)$ satisfy the assumptions of Theorem 4(i), we note that since $Z_{n} e^{i \Phi_{n}}$ are independent symmetric, it suffices to prove the a.s. unboundedness of the random Fourier series

$$
\sum_{1}^{\infty} Z_{n} \varepsilon_{n} \sin \left(n t+\Phi_{n}\right)
$$

where $\left\{\varepsilon_{n}\right\}$ is a Rademacher sequence independent of the sequence $\left\{\left(Z_{n}, \Phi_{n}\right)\right\}$. Let $\tilde{Z}_{n}=Z_{n} \varepsilon_{n}$. Then $\tilde{Z}_{n}$ is symmetric and the assumptions (3.6)-(3.8) still hold with $\tilde{Z}_{n}$ replacing $Z_{n}$ (see Remark (i)). Hence we can assume without loss of generality that $Z_{n}$ is symmetric. Making this assumption and using the same argument as in [10, p. 77], we can reduce the problem of a.s. unboundedness of (4.1) to the a.s. unboundedness of

$$
\sum_{1}^{\infty} Z_{n} \sin \left(n t+\Psi_{n}\right)
$$

where $\left\{\Psi_{n}\right\}$ is an i.i.d. sequence of random variables uniformly distributed in $[0,2 \pi]$ and independent of the sequence $\left\{\left(Z_{n}, \Phi_{n}\right)\right\}$. Since we have already established the a.s. unboundedness of (3.25) with $Z_{n}$ symmetric, the proof is complete.

Proof of Theorem 4(ii). Let $\left\{Z_{n}^{*}\right\}$ be a sequence of independent random variables such that $\left\{Z_{n}\right\}$ and $\left\{Z_{n}^{*}\right\}$ are independent and $Z_{n}^{*}$ has the same distribution as $Z_{n}$ for every $n$. Note that $E\left|\sum_{n=l}^{m} Z_{n}\right|<E\left|\sum_{n=1}^{m}\left(Z_{n}-Z_{n}^{*}\right)\right|$ since $\left\{Y_{1}=\right.$ $\left.\sum_{n=l}^{m} Z_{n}, Y_{2}=\sum_{n=1}^{m}\left(Z_{n}-Z_{n}^{*}\right)\right\}$ forms a martingale. Moreover, $\left(Z_{n}-Z_{n}^{*}\right) e^{i \Phi_{n}}$ are independent symmetric. Hence by Theorem 4(i), (3.2), (3.9), and (3.10) hold when $Z_{n}$ is replaced by $Z_{n}-Z_{n}^{*}$. By this implies that (3.2), (3.9), and (3.10) have to hold (for $Z_{n}$ ) since $\left\{Z_{n}\right\}$ and $\left\{Z_{n}^{*}\right\}$ have the same distribution.

We now apply Theorem 4 to the random Fourier series (1.1) where the $X_{n}$ are i.i.d. 
THEOREM 5. Let $\left\{a_{n}\right\} \in l^{2}$ and assume that $X$ is symmetric and nondegenerate. Suppose that there exists $\psi:[0, \infty) \rightarrow\{1,2, \ldots\}$ such that

$$
\begin{aligned}
& E\left\{X^{2}\left(\sum_{n=\psi(|X|)}^{\infty} a_{n}^{2}\right)\right\}<\infty, \text { and } \\
& \sum_{k=1}^{\infty}\left\{\min _{2^{k}<n<2^{k+1}}\left|a_{n}\right|\right\} G_{\psi}\left(2^{k}\right)=\infty,
\end{aligned}
$$

where $G_{\psi}(n)>0$ is the unique solution of the equation

$$
\left.n E\left(\min \left\{\left(X I_{[\psi(|X|)} \leqslant n\right] / G_{\psi}(n)\right)^{2},\left|X I_{[\psi(|X|)<n]}\right| / G_{\psi}(n)\right\}\right)=1
$$

if $X I_{[\psi(|X|)<n]}$ is nondegenerate, and $G_{\psi}(n)=0$ otherwise. Then (3.2), (3.9), and (3.10) hold with $Z_{n}=a_{n} X_{n}$.

REMARK. Suppose that $\left|a_{n}\right| \downarrow$ and that $\lim _{\inf }{ }_{n \rightarrow \infty}\left|a_{2 n}\right| /\left|a_{n}\right|>0$. If $0<E X^{2}=\sigma^{2}$ $<\infty$, then (3.26) holds and $G_{\psi}(n) \sim \sigma n^{1 / 2}$ for $\psi \equiv 1$; therefore with this choice of $\psi$ and under the above regularity conditions on $\left\{a_{n}\right\},(3.27)$ is equivalent to

$$
\sum_{k=1}^{\infty}\left(\sum_{n=2^{k}}^{2^{k+1}-1} a_{n}^{2}\right)^{1 / 2}=\infty
$$

which is in turn equivalent to the divergence of the integral in (1.2) under the further assumption that $\sum_{n=2^{k}}^{2^{k+1}} a_{n \downarrow}^{2} \downarrow$ (cf. [10, p. 64] and Remark 2.7 of [20]).

The function $G_{\psi}$ defined by (3.28) is related to the following result of Klass [11, p. 166]:

LemMA 6. Let $Y, Y_{1}, Y_{2}, \ldots$ be i.i.d. random variables such that $E Y=0$ and $E|Y|>0$. Let $S_{n}=\sum_{1}^{n} Y_{i}$. For $y \geqslant 1$, let $G(y)$ be the unique solution of

$$
y E\left(\min \left\{(Y / G(y))^{2},|Y / G(y)|\right\}\right)=1 \text {. }
$$

Then $E\left|S_{n}\right|=2 E S_{n}^{+}$and

$$
\frac{1}{3} G(n) \leqslant E\left|S_{n}\right| \leqslant 2 G(n)
$$

Proof of Theorem 5. Let $X_{n}^{\prime}=X_{n} I_{\left[\psi\left(\left|X_{n}\right|\right)<2^{k}\right]}$ for $2^{k}<n<2^{k+1}$, and let $X_{1}^{\prime}=0$. By (3.26),

$$
\sum_{1}^{\infty} E\left(a_{n} X_{n}^{\prime}\right)^{2}<\sum_{1}^{\infty} a_{n}^{2} E X^{2} I_{[\psi(|X|)<n]}=E\left\{X^{2}\left(\sum_{n=\psi(|X|)}^{\infty} a_{n}^{2}\right)\right\}<\infty .
$$

By Lemma 4,

$$
\begin{aligned}
E\left|\sum_{n=2^{k}}^{2^{k+1}-1} a_{n} X_{n}^{\prime}\right| & \geqslant\left(\min _{2^{k}<n<2^{k+1}}\left|a_{n}\right|\right) E\left|\sum_{n=2^{k}}^{2^{k+1}-1} X_{n}^{\prime}\right| \\
& \geqslant \frac{1}{3}\left(\min _{2^{k}<n<2^{k+1}}\left|a_{n}\right|\right) G_{\psi}\left(2^{k}\right) \text { for all large } k .
\end{aligned}
$$

The last inequality follows from Lemma 6 , noting that $\left\{X_{n}^{\prime}: 2^{k}<n<2^{k+1}\right\}$ is a set of $2^{k}$ i.i.d. symmetric random variables. Hence (3.27) implies that 


$$
\sum_{k=1}^{\infty} E\left|\sum_{n=2^{k}}^{2^{k+1}-1} a_{n} X_{n}^{\prime}\right|=\infty
$$

In view of (3.31) and (3.32), it follows from Theorem 5 that (3.2) holds with $Z_{n}$ replaced by $a_{n} X_{n}^{\prime}$. By the generalized contraction principle of Jain and Marcus [8, Theorem 5.1], this in turn implies that (3.2) holds with $Z_{n}=a_{n} X_{n}$ in the case $\Phi_{n} \equiv 0$, and the result then extends to the general $\Phi_{n}$ (which are independent of the symmetric random variables $X_{n}$ ) by a theorem of Marcus [18, Corollary 3.2].

As an application of Theorem 5, we now give the proof of Theorem 1(vi), (vii) in the following

COROllary 3. (i) Let $\frac{1}{2}<\alpha<1$, and let $X$ have density

$$
\begin{aligned}
f_{\alpha}(x) & =C_{\alpha} /\left\{|x|(|x| \log |x|)^{1 / \alpha}\right\}, \quad|x|>e, \\
& =0, \quad|x|<e,
\end{aligned}
$$

where $C_{\alpha}$ is chosen so that $\int_{-\infty}^{\infty} f_{\alpha}(x) d x=1$. Then $X$ is symmetric and

$$
\begin{aligned}
E\left\{|X|^{1 / \alpha}\left(\log ^{+}|X|\right)^{\gamma}\right\} & <\infty \quad \text { if } \gamma<(1-\alpha) / \alpha, \\
& =\infty \quad \text { if } \gamma>(1-\alpha) / \alpha .
\end{aligned}
$$

Moreover, (3.2), (3.9), and (3.10) hold with $Z_{n}=n^{-\alpha} X_{n}$. Hence with probability 1, $\Sigma_{1}^{\infty} n^{-\alpha} X_{n} \sin \left(n t+\Phi_{n}\right)$ converges a.e. but not everywhere.

(ii) Let $1<\beta \leqslant 2$, and let $X$ have density

$$
\begin{aligned}
f(x) & =C /\left\{x^{2}(\log |x|)(\log \log |x|)^{\beta}\right\}, \quad|x|>e^{e}, \\
& =0, \quad|x|<e^{e}
\end{aligned}
$$

where $C$ is chosen so that $\int_{-\infty}^{\infty} f(x) d x=1$. Then $X$ is symmetric and

$$
\begin{array}{rlrl}
E\left\{|X|[\log \log (|X|+e)]^{\gamma}\right\} & <\infty & & \text { if } \gamma<\beta-1, \\
& =\infty & \text { if } \gamma>\beta-1 .
\end{array}
$$

Moreover, (3.2), (3.9), and (3.10) hold with $Z_{n}=n^{-1} X_{n}$. Hence with probability 1, $\Sigma_{1}^{\infty} n^{-1} X_{n} \sin \left(n t+\Phi_{n}\right)$ converges a.e. but not everywhere.

Proof. Let $a_{n}=n^{-\alpha}$, and let $\psi(x)$ be the smallest integer $>x^{1 / \alpha}$ if $x>0$, setting $\psi(0)=1$. Since $E|X|^{1 / \alpha}<\infty,(3.26)$ holds and it follows from Theorem 1(i) that $P[S(t)$ converges a.e. $]=1$. Defining $G_{\psi}(n)$ as in (3.28) and making use of the density functions (3.33) and (3.34), it is easy to show that

$$
\begin{aligned}
G_{\psi}(n) & \sim D_{\alpha} n^{\alpha} /(\log n) \quad \text { for } \alpha<1 \text { (i.e., the case (3.33)), } \\
& \sim D_{\alpha} n /\left\{(\log n)(\log \log n)^{\beta-1}\right\} \quad \text { for } \alpha=1 \text { (i.e., the case (3.34)), }
\end{aligned}
$$

where $D_{\alpha}$ is a positive constant. Noting that $\beta-1<1$, we obtain (3.27) from (3.35). Therefore by Theorem 5, (3.2), (3.9), and (3.10) hold with $Z_{n}=n^{-\alpha} X_{n}$.

4. Law of the iterated logarithm related to local behavior. In this section we shall assume that $E X=0$ and $E X^{2}<\infty$, and we shall study the local behavior of the series (1.1) with

$$
a_{n}=O\left(n^{-\alpha}\right)
$$


for some $\alpha>\frac{1}{2}$. An obvious modification of the proof in $\$ 2$ shows that parts (ii)-(v) and (viii) of Theorem 1 can be extended to cover the case (4.1). Hence $S(\cdot)$ is a.s. continuous, and in the case $\alpha>3 / 2, S(\cdot)$ is a.s. continuously differentiable. A useful tool in our analysis is the following well-known bound, due to Salem and Zygmund [25], for the maximum of a random trigonometric polynomial.

LEMMA 7. Let $k$ be a positive integer and let $U_{0}, U_{1}, \ldots, V_{0}, V_{1}, \ldots$ be random variables. Let $P(t)=\sum_{0}^{k} U_{n} \sin \left(n t+V_{n}\right)$ be a random trigonometric polynomial of degree $<k$. Let $M=\max _{t}|P(t)|$. Then there exists a random interval $I$ of length not less than $(2 k)^{-1}$ such that $\min _{t \in I}|P(t)|>\frac{1}{2} M$. Consequently for every $\lambda>0$,

$$
E e^{\lambda M / 2}<2 k \int_{0}^{2 \pi}\left(E e^{\lambda P(t)}+E e^{-\lambda P(t)}\right) d t .
$$

In the case where $X$ is subnormal (i.e., $E e^{\lambda X}<\exp \left(\frac{1}{2} \lambda^{2}\right)$ for all $\lambda$ ), Kahane [9] made use of the above Salem-Zygmund bound to estimate the order of magnitude of the modulus of continuity of $S(\cdot)$. By using a truncation technique together with a modification of Kahane's argument, we can remove the subnormal assumption on $X$ in the following

THEOREM 6. Suppose that $\left\{a_{n}\right\}$ satisfies (4.1) for some $\frac{1}{2}<\alpha<\frac{3}{2}$ and that EX $=0$ and $E X^{2}<\infty$. Then with probability 1 ,

$$
\sup _{\left|t-t^{\prime}\right|<h}\left|S(t)-S\left(t^{\prime}\right)\right|=O\left(\left\{h^{2 \alpha-1}|\log h|\right\}^{1 / 2}\right) \quad \text { as } h \downarrow 0 \text {. }
$$

Proof. Let $\sigma^{2}=E X^{2}$. Define

$$
\begin{aligned}
& X_{n}^{\prime}=X_{n} I_{\left[\left|X_{n}\right|<n^{1 / 2}(\log 2 n)^{-1 / 2}\right]}-E X I_{\left[|X|<n^{1 / 2}(\log 2 n)^{-1 / 2}\right]}, \\
& X_{n}^{\prime \prime}=X_{n} I_{\left[\left|X_{n}\right|>n^{1 / 2}(\log 2 n)^{-1 / 2}\right]} .
\end{aligned}
$$

We note that

$$
\begin{aligned}
S(t)= & \sum_{1}^{\infty} a_{n} X_{n}^{\prime} \sin \left(n t+\Phi_{n}\right)+\sum_{1}^{\infty} a_{n} X_{n}^{\prime \prime} \sin \left(n t+\Phi_{n}\right) \\
& +\sum_{1}^{\infty} a_{n}\left(E X I_{\left[|X|<n^{1 / 2}(\log 2 n)^{-1 / 2}\right]}\right) \sin \left(n t+\Phi_{n}\right) \\
= & S_{1}(t)+S_{2}(t)+S_{3}(t), \text { say. }
\end{aligned}
$$

Since $\left|E X I_{\left[|X|>n^{1 / 2}(\log 2 n)^{-1 / 2}\right]}\right| \leqslant\left(E X^{2}\right)(\log 2 n)^{1 / 2} / n^{1 / 2}$, and since

$$
|\sin (x+y)-\sin x|<2 \min (1,|y|) \text {, }
$$

we obtain that for $\left|t-t^{\prime}\right| \leqslant h$

$$
\begin{aligned}
\left|S_{3}(t)-S_{3}\left(t^{\prime}\right)\right| & \leqslant 2\left(E X^{2}\right)\left\{\sum_{n<h^{-1}} n^{1 / 2} h\left|a_{n}\right|(\log 2 n)^{1 / 2}+\sum_{n>h^{-1}}\left|a_{n}\right|(\log 2 n)^{1 / 2} / n^{1 / 2}\right\} \\
& =O\left(h^{\alpha-1 / 2}|\log h|^{1 / 2}\right), \text { by (4.1). }
\end{aligned}
$$

The finiteness of $E X^{2}$ implies that

$$
\sum_{1}^{\infty}(n \log 2 n)^{-1 / 2} E\left|X_{n}^{\prime \prime}\right|=E\left\{|X| \sum_{n^{1 / 2}(\log 2 n)^{-1 / 2}<|X|}(n \log 2 n)^{-1 / 2}\right\}<\infty,
$$


and therefore $\Sigma_{2}^{\infty}(n \log n)^{-1 / 2}\left|X_{n}^{\prime \prime}\right|<\infty$ a.s. In view of (4.1), this in turn implies that with probability 1 ,

$$
\begin{gathered}
m^{\alpha-1 / 2}(\log m)^{-1 / 2} \sum_{n=m}^{\infty}\left|a_{n} X_{n}^{\prime \prime}\right|=O(1), \text { and } \\
\sum_{n=1}^{m} n\left|a_{n} X_{n}^{\prime \prime}\right|=o\left(m^{3 / 2-\alpha}(\log m)^{1 / 2}\right) \quad \text { by the Kronecker lemma. }
\end{gathered}
$$

From (4.3), (4.5), and (4.6), it follows that with probability 1

$$
\begin{aligned}
\sup _{\left|t-t^{\prime}\right|<h}\left|S_{2}(t)-S_{2}\left(t^{\prime}\right)\right| & \leqslant 2\left\{h \sum_{n<h^{-1}} n\left|a_{n} X_{n}^{\prime \prime}\right|+\sum_{n>h^{-1}}\left|a_{n} X_{n}^{\prime \prime}\right|\right\} \\
& =O\left(h^{\alpha-1 / 2}|\log h|^{1 / 2}\right) .
\end{aligned}
$$

We now consider $S_{1}(t)$. Given a positive integer $m$, define $\nu_{0}=0, \nu_{k}=m 2^{k-1}$, $N_{k}=2^{v_{k}}$, and

$$
\begin{aligned}
P_{k}(t) & =\sum_{N_{k}<n<N_{k+1}} a_{n} X_{n}^{\prime} \sin \left(n t+\Phi_{n}\right), \\
\lambda_{k} & =\delta\left\{\left(\log N_{k+1}\right) /\left(\sum_{N_{k}<n<N_{k+1}} n^{-2 \alpha}\right)\right\}^{1 / 2},
\end{aligned}
$$

where $\delta$ is a positive constant (not depending on $m$ and $k$ ) to be specified later. In view of (4.1) and the bound $\left|X_{n}^{\prime}\right|<2 n^{1 / 2}(\log 2 n)^{-1 / 2}$, we can choose $\delta$ sufficiently small so that

$$
\left(\lambda_{k} a_{n} X_{n}^{\prime}\right)^{2}<1 \text { for } N_{k}<n<N_{k+1}, k=1,2, \ldots
$$

If $Z$ is a random variable such that $E Z=0$ and $|Z|<1$, then it is well known that

$$
E \exp (t Z)<\exp \left\{\frac{1}{2} t^{2}\left(E Z^{2}\right)\left(1+\frac{1}{2}|t|\right)\right\} \text { for }|t|<1
$$

(cf. [15, p. 255]). In view of (4.1), (4.8), (4.9), and the fact that $E\left\{X_{n}^{\prime} \sin \left(n t+\Phi_{n}\right)\right\}^{2}$ $\leqslant \sigma^{2}$, we can choose $\delta$ sufficiently small so that

$$
\begin{aligned}
E \exp \left\{\lambda_{k} P_{k}(t)\right\} & =\prod_{N_{k}<n<N_{k+1}} E \exp \left\{\lambda_{k} a_{n} X_{n}^{\prime} \sin \left(n t+\Phi_{n}\right)\right\} \\
& <\exp \left(\frac{1}{2} \log N_{k+1}\right) \text { for all } k \text { and } t .
\end{aligned}
$$

Replacing $X_{n}^{\prime}$ by $-X_{n}^{\prime}$ in (4.10), we also obtain that

$$
E \exp \left\{-\lambda_{k} P_{k}(t)\right\}<\exp \left(\frac{1}{2} \log N_{k+1}\right)
$$

Let $M_{k}=\max _{t}\left|P_{k}(t)\right|$. It then follows from (4.10), (4.11), and Lemma 7 that

$$
\begin{aligned}
E \exp \left(\frac{1}{2} \lambda_{k} M_{k}\right) & \leqslant 2 N_{k+1} \int_{0}^{2 \pi} E\left(e^{\lambda_{k} P_{k}(t)}+e^{-\lambda_{k} P_{k}(t)}\right) d t \\
& <8 \pi \exp \left(\frac{3}{2} \log N_{k+1}\right) .
\end{aligned}
$$

In view of (4.12), we can choose a sufficiently large positive constant $C$ (not depending on $m$ and $k$ ) such that for all $k>1$, 


$$
\begin{aligned}
P\left[\max _{t}\left|P_{k}(t)\right|\right. & \left.\geqslant C\left(\log N_{k+1}\right)^{1 / 2}\left(\sum_{N_{k}<n<N_{k+1}} n^{-2 \alpha}\right)^{1 / 2}\right] \\
& \leqslant \exp \left\{-\frac{1}{2} \delta C\left(\log N_{k+1}\right)\right\} E \exp \left(\frac{1}{2} \lambda_{k} M_{k}\right)<N_{k+1}^{-2} .
\end{aligned}
$$

Noting that $P_{0}^{\prime}(t)=-\sum_{1}^{N_{1}-1} n a_{n} X_{n}^{\prime} \sin \left(n t+\Phi_{n}-\frac{1}{2} \pi\right)$, we can use a similar argument and choose $C$ sufficiently large so that

$$
P\left[\max _{t}\left|P_{0}^{\prime}(t)\right|>C\left(\log N_{1}\right)^{1 / 2}\left(\sum_{n<N_{1}} n^{2-2 \alpha}\right)^{1 / 2}\right]<N_{1}^{-2} .
$$

By the argument of Kahane [10, pp. 66-68], the estimates (4.13) and (4.14) can in turn be used to show that with probability 1

$$
\sup _{\left|t-t^{\prime}\right|<h}\left|S_{1}(t)-S_{1}\left(t^{\prime}\right)\right|=O\left(h^{\alpha-1 / 2}|\log h|^{1 / 2}\right)
$$

From (4.4), (4.7), and (4.15), the desired conclusion (4.2) follows.

While the modulus of continuity $\sup _{\left|t-t^{\prime}\right|<h}\left|S(t)-S\left(t^{\prime}\right)\right|$ can be effectively estimated by the preceding argument involving the Salem-Zygmund bound for the maximum of a random trigonometric polynomial, this approach fails to handle the more delicate problem of the law of the iterated logarithm related to the local fluctuation of $S(t)-S\left(t_{0}\right)$ as $t \rightarrow t_{0}$. Instead of a single logarithmic term as in (4.2), the local behavior of $S(t)$ as $t \rightarrow t_{0}$ involves a $\log \log$ term as in (1.8). To establish such iterated logarithm results, we shall use the Salem-Zygmund bound in another way together with an embedding technique to reduce the problem to the Gaussian case where we are able to obtain precise estimates of the local fluctuation of the Gaussian process by proving certain properties of its covariance function.

We first note that

$$
\begin{aligned}
S\left(t_{0}+h\right)-S\left(t_{0}\right)= & \sum_{1}^{\infty} a_{n} X_{n}\left\{\sin \left(n t_{0}+n h+\Phi_{n}\right)-\sin \left(n t_{0}+\Phi_{n}\right)\right\} \\
= & \sum_{1}^{\infty} a_{n}\left\{X_{n} \sin \left(n t_{0}+\Phi_{n}\right)\right\}(\cos n h-1) \\
& +\sum_{1}^{\infty} a_{n}\left\{X_{n} \cos \left(n t_{0}+\Phi_{n}\right)\right\} \sin n h \\
= & U_{1}(h)+U_{2}(h), \text { say. }
\end{aligned}
$$

Hence if it can be shown that

$$
P\left[U_{i}(h)=O\left(|h|^{\alpha-1 / 2}\left(\log \log |h|^{-1}\right)^{1 / 2}\right) \text { as } h \rightarrow 0\right]=1
$$

for $i=1,2$, then

$$
S\left(t_{0}+h\right)-S\left(t_{0}\right)=O\left(|h|^{\alpha-1 / 2}\left(\log \log |h|^{-1}\right)^{1 / 2}\right) \quad \text { a.s. }
$$


Since $\cos n h-1=\sin (-\pi / 2)-\sin (-\pi / 2+n h)$, both $U_{1}(h)$ and $U_{2}(h)$ can be written in the form $Z(h)-Z(0)$, where

$$
Z(t)=\sum_{1}^{\infty} b_{n} Y_{n} \sin (n t+\theta)
$$

and $b_{n}, \theta$ are constants and $\left\{Y_{n}\right\}$ is sequence of independent zero-mean random variables. The following theorem gives conditions on $b_{n}$ and $Y_{n}$ for the law of the iterated logarithm to hold for $Z(t)$ as $t \rightarrow 0$. It also includes Theorem 2 as a special case and gives the law of the iterated logarithm (1.8) in the stronger form of an integral test.

THEOREM 7. Suppose that $Y_{1}, Y_{2}, \ldots$ are independent random variables such that $E Y_{n}=0, E Y_{n}^{2}=\sigma_{n}^{2}<\infty$ for all $n$, and

$$
\liminf _{n \rightarrow \infty} n^{-1} \sum_{1}^{n} \sigma_{k}^{2}>0 \text {. }
$$

Let $\frac{1}{2}<\alpha<\frac{3}{2}$, and assume that

$$
\sup _{n} E\left\{Y_{n}^{2}\left(\log ^{+}\left|Y_{n}\right|\right)^{p}\right\}<\infty
$$

for some $p>(6 \alpha+1) /(2 \alpha-1)$. Let $b_{n}, \theta$ be constants and define the random Fourier series $Z(t)$ as in (4.17). Let

$$
v_{s}(t)=E(Z(t+s)-Z(s))^{2}=\sum_{1}^{\infty} b_{n}^{2} \sigma_{n}^{2}\{\sin (n(t+s)+\theta)-\sin (n s+\theta)\}^{2}
$$

Assume that

$$
\sum_{k=n}^{\infty}\left|b_{k}-b_{k+1}\right|=O\left(n^{-\alpha}\right),
$$

and that there exist positive constants $c$ and $\delta$ such that

$$
v_{s}(t) \geqslant c|t|^{2 \alpha-1} \text { for all }|t|<\delta \text { and }|s|<\delta .
$$

Let $g$ be a positive nonincreasing function on $(0, a]$ with $a>0$. Then

$$
P\left[|Z(t)-Z(0)|<v_{0}^{1 / 2}(t) g(|t|) \text { for all small }|t|>0\right]=1 \text { or } 0
$$

according as

$$
\int_{0+}^{a} t^{-1}(g(t))^{(3-2 \alpha) /(2 \alpha-1)} \exp \left\{-\frac{1}{2} g^{2}(t)\right\} d t<\infty \text { or }=\infty .
$$

The proof of Theorem 7 depends on the following lemmas.

LEMMA 8. Let $\{U(t),|t|<1\}$ be a real separable Gaussian process such that $E U(t)=0$ for all $t$. Define

$$
\sigma_{s}^{2}(t)=E(U(s+t)-U(s))^{2} .
$$

Assume that there exist positive constants $\beta<2, \frac{1}{2}<\gamma<1, c_{1}<c_{2}$, and $\delta$ such that for all $|t|<\delta,|s|<\delta$ and $|h|<\delta$, 


$$
\begin{aligned}
\sigma_{s}^{2}(t) & >c_{1}|t|^{\beta} \text {, and } \\
\left|\sigma_{s}^{2}(t+h)-\sigma_{s}^{2}(h)\right| & <c_{2}|t|^{\gamma \beta}(\max \{|t|,|h|\})^{(1-\gamma) \beta} .
\end{aligned}
$$

(i) Let $g$ be a positive nonincreasing function on $(0, a]$. Then

$$
\begin{aligned}
& P\left[|U(t)-U(0)|<\sigma_{0}(t) g(|t|) \text { for all small }|t|>0\right]=1 \text { or } 0 \\
& \text { according as } I(g)<\infty \text { or }=\infty,
\end{aligned}
$$

where $I(g)=\int_{0+}^{a} t^{-1}(g(t))^{(2 / \beta)-1} \exp \left\{-\frac{1}{2} g^{2}(t)\right\} d t$.

(ii) Let $Z(t)=Z(0)+U(t)-U(0)+R(t)$, where $\{R(t),|t|<1\}$ is a stochastic process such that with probability 1

$$
R(t)=o\left(|t|^{\beta / 2}\left(\log \log |t|^{-1}\right)^{-1 / 2}\right) \quad \text { as } t \rightarrow 0 .
$$

Then (4.28) still holds when $U(\cdot)$ is replaced by $Z(\cdot)$.

Proof. To prove (4.28), we shall restrict to the case $t>0$, as the case $t<0$ is obviously analogous. Since $\sigma_{s}^{2}(0)=0,(4.27)$ implies that

$$
\sigma_{s}^{2}(t)<c_{2}|t|^{\beta} \quad \text { for all }|t|<\delta \text { and }|s|<\delta \text {. }
$$

We shall use the following transformation of Nisio [22, p. 320]:

$$
\begin{aligned}
Y(n+t)=\left\{U\left(2^{-n}-t 2^{-n-1}\right)-U(0)\right\} / \sigma_{0}\left(2^{-n}-t 2^{-n-1}\right), & \\
0 & <t<1, n=1,2, \ldots .
\end{aligned}
$$

Clearly $E Y(t)=0$ and $E Y^{2}(t)=1$. For $n>n_{0}$ (sufficiently large) and $s, t \in[0,1]$,

$$
E\{Y(n+t)-Y(n+s)\}^{2}=E(W-V)^{2}
$$

where

$$
\begin{aligned}
W= & \left\{U\left(2^{-n}-t 2^{-n-1}\right)-U\left(2^{-n}-s 2^{-n-1}\right)\right\} / \sigma_{0}\left(2^{-n}-t 2^{-n-1}\right), \\
V= & \left\{U\left(2^{-n}-s 2^{-n-1}\right)-U(0)\right\} \\
& \cdot\left\{\left(\sigma_{0}\left(2^{-n}-s 2^{-n-1}\right)\right)^{-1}-\left(\sigma_{0}\left(2^{-n}-t 2^{-n-1}\right)\right)^{-1}\right\}
\end{aligned}
$$

and by (4.26) and (4.30),

$$
\left(c_{1} / c_{2}\right)|t-s|^{\beta}<E W^{2}<\left(c_{2} / c_{1}\right)|t-s|^{\beta}
$$

moreover, by (4.26) and (4.27),

$$
\begin{aligned}
E V^{2} & =\frac{\left\{\sigma_{0}^{2}\left(2^{-n}-t 2^{-n-1}\right)-\sigma_{0}^{2}\left(2^{-n}-s 2^{-n-1}\right)\right\}^{2}}{\left\{\sigma_{0}\left(2^{-n}-t 2^{-n-1}\right)+\sigma_{0}\left(2^{-n}-s 2^{-n-1}\right)\right\}^{2} \sigma_{0}^{2}\left(2^{-n}-t 2^{-n-1}\right)} \\
& \leqslant c_{2}^{2}\left(2^{-n-1}|t-s|\right)^{2 \gamma \beta} 2^{-2(1-\gamma) \beta n} /\left\{c_{1}^{2} 2^{-2 \beta(n+1)}\right\} \\
& <\left(2^{\beta} c_{2} / c_{1}\right)^{2}|t-s|^{2 \gamma \beta} .
\end{aligned}
$$

Since $2\left(E W^{2}+E V^{2}\right) \geqslant E(W-V)^{2}>E W^{2}-2\left(E W^{2}\right)^{1 / 2}\left(E V^{2}\right)^{1 / 2}$ and since $2 \gamma$ $>1$, it then follows from (4.32)-(4.34) that there exist positive constants $C_{2}>C_{1}$ and $\delta^{*}$ such that for all $n>n_{0}$ and $s, t \in[0,1]$ with $|t-s|<\delta^{*}$,

$$
C_{1}|t-s|^{\beta}<E\{Y(n+t)-Y(n+s)\}^{2}<C_{2}|t-s|^{\beta} \text {. }
$$


For $u, v \in[0,1]$ and $n \geqslant n_{0}$, noting that

$$
2^{-n-1}(u+v) \leqslant\left\{2^{-n+1}-(1-v) 2^{-n}\right\}-\left\{2^{-n}-u 2^{-n-1}\right\}<2^{-n}(u+v),
$$

a similar argument as before shows that for $u+v<\delta^{*}$,

$$
C_{1}(u+v)^{\beta} \leqslant E\{Y(n+u)-Y((n-1)+(1-v))\}^{2}<C_{2}(u+v)^{\beta} .
$$

Hence for all $t, s \geqslant n_{0}+1$ such that $|t-s|<\delta^{*}$,

$$
C_{1}|t-s|^{\beta} \leqslant E(Y(t)-Y(s))^{2} \leqslant C_{2}|t-s|^{\beta} .
$$

Using the identity $2 \operatorname{Cov}(X, Y)=\operatorname{Var} X+\operatorname{Var} Y-\operatorname{Var}(X-Y)$, we obtain that for $m \geqslant n \geqslant n_{0}$ and $s, t \in[0,1]$,

$$
\begin{aligned}
& 2\left|E\left\{\left(U\left(2^{-n}-t 2^{-n-1}\right)-U(0)\right)\left(U\left(2^{-m}-s 2^{-m-1}\right)-U(0)\right)\right\}\right| \\
& =\mid \sigma_{0}^{2}\left(2^{-m}-s 2^{-m-1}\right)+\sigma_{2^{-n}-t 2^{-n-1}}\left(-2^{-n}+t 2^{-n-1}\right)
\end{aligned}
$$

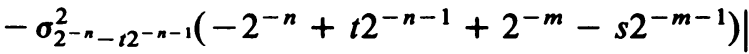

$$
\begin{aligned}
& \leqslant c_{2}\left(2^{-m \beta}+2^{-m \gamma \beta-n(1-\gamma) \beta}\right), \quad \text { by (4.27) and (4.30). }
\end{aligned}
$$

From (4.26) and (4.36), it then follows that for $m>n>n_{0}$ and $s, t \in[0,1]$,

$$
|E\{Y(n+t) Y(m+s)\}| \leqslant\left(2^{1+\beta} c_{2} / c_{1}\right) 2^{-(\gamma-1 / 2) \beta(m-n)} .
$$

In view of (4.35) and (4.37), we obtain by a theorem of Qualls and Watanabe [24, pp. 2034-2035] that if $h$ is a positive nondecreasing function on $[1, \infty)$ then

$$
P[|Y(u)|<h(u) \text { for all large } u]=1 \text { or } 0
$$

according as

$$
\int_{1}^{\infty}(h(u))^{(2 / \beta)-1} \exp \left\{-\frac{1}{2} h^{2}(u)\right\} d u<\infty \text { or }=\infty .
$$

Applying the change of variable $\tau=\tau(u)=2^{-n}-2^{-n-1}(u-n)$ for $n<u<n+$ $1(n=1,2, \ldots)$ to the integral above and defining $g(\tau)=h(u)$, we obtain (4.28) with $t$ restricted to positive values.

To prove (ii), we first note that by (4.26) and (4.29), $R(t) / \sigma_{0}(t)=$ $o\left(\left(\log \log |t|^{-1}\right)^{-1 / 2}\right)$ a.s. A standard argument (see for example the proof of Theorem 5.1 of [6] and Lemma 1.4 of [24]) first reduces the problem to the case where $\left(\log \log t^{-1}\right)^{1 / 2} \leqslant g(t) \leqslant 2\left(\log \log t^{-1}\right)^{1 / 2}$ so that $R(t) / \sigma_{0}(t)=o(1 / g(t))$ a.s., and then proves (ii) from (i) under this additional assumption on $g$.

RemarK. For the special case where $U(t)$ is stationary, Sirao and Watanabe [26, Theorems 4, 5] have established Lemma 8(i) under the further assumption that $\sigma_{0}^{2}(t)$ is concave and nondecreasing for $t>0$. This assumption of concavity and monotonicity of $\sigma_{0}^{2}(t)$ is rather stringent in view of our applications, and we are able to replace it by the assumption (4.27) using the argument above.

Lemma 9. Let $\gamma>0$. Suppose that $Y_{1}, Y_{2}, \ldots$ are independent random variables such that $E Y_{n}=0$ for all $n$ and (4.19) holds for $p>4(1+\gamma)+1$. Letting $E Y_{n}^{2}=$ $\sigma_{n}^{2}$, assume that (4.18) holds. Then redefining the $Y_{n}$ on a new probability space if necessary, there exist independent normal random variables $\xi_{1}, \xi_{2}, \ldots$ on the same probability space such that $\xi_{n}$ has mean 0 and variance $\sigma_{n}^{2}(n=1,2, \ldots)$ and

$$
\left(\sum_{1}^{n} Y_{i}-\sum_{1}^{n} \xi_{i}\right) /\left\{n^{1 / 2}(\log n)^{-\gamma}\right\} \rightarrow 0 \text { a.s. }
$$


Proof. Let $f(t)=t /(\log t)^{4(1+\gamma)}$, and let $v_{n}=\Sigma_{1}^{n} \sigma_{i}^{2}$. The conditions (4.18) and (4.19) imply that

$$
\begin{gathered}
c_{1} n \leqslant v_{n} \leqslant c_{2} n \quad \text { for some } c_{2}>c_{1}>0 \text { and all } n \text {, and } \\
\sum_{n=m}^{\infty}\left(f\left(v_{n}\right)\right)^{-1} E\left\{Y_{n}^{2} I_{\left[Y_{n}^{2}>f\left(v_{n}\right)\right]}\right\}<\infty .
\end{gathered}
$$

Hence by a theorem of Strassen [27, Theorem 4.4], there exists a standard Wiener process $W(t)$ such that (redefining the random variables on a new probability space if necessary) with probability 1

$$
\sum_{1}^{n} Y_{i}=W\left(v_{n}\right)+o\left(\left(\log v_{n}\right)\left(v_{n} f\left(v_{n}\right)\right)^{1 / 4}\right)
$$

Setting $\xi_{n}=W\left(v_{n}\right)-W\left(v_{n-1}\right)\left(v_{0}=0\right)$, the desired conclusion (4.38) follows from (4.39) and (4.41).

LEMMA 10. Let $\left\{b_{n}\right\}$ be a sequence of constants such that $b_{n}=O\left(n^{-\alpha}\right)$ for some $\alpha>\frac{1}{2}$. Let $\left\{Y_{n}\right\}$ be a sequence of independent random variables such that $E Y_{n}=0$ for all $n$ and (4.19) holds for some $p>1$. Let $\left\{\Phi_{n}\right\}$ be a sequence of independent random variables which are also independent of the sequence $\left\{Y_{n}\right\}$. Then with probability 1,

$$
\sup _{t}\left|\sum_{2^{k}<n} b_{n} Y_{n} \sin \left(n t+\Phi_{n}\right)\right|=O\left(k^{1 / 2} 2^{-k(\alpha-1 / 2)}\right)
$$

Proof. Define $Y_{n}^{\prime \prime}=Y_{n} I_{\left[\left|Y_{n}\right|>(n / \log n)^{1 / 2}\right]}$ and let $Y_{n}^{\prime}=Y_{n}-Y_{n}^{\prime \prime}-E Y_{n}^{\prime \prime}$. Using the assumption that (4.19) holds for some $p>1$, it is easy to show that

$$
\begin{gathered}
\sum_{2}^{\infty}(n \log n)^{-1 / 2} E\left|Y_{n}^{\prime \prime}\right|<\infty, \text { and } \\
\sum_{n=m}^{\infty}\left|b_{n}\right|\left|E Y_{n}^{\prime \prime}\right|=O\left(\sum_{n=m}^{\infty} n^{-\alpha-1 / 2}\right)=O\left(m^{-\alpha+1 / 2}\right) .
\end{gathered}
$$

By (4.43), the fact that $b_{n}=O\left(n^{-\alpha}\right)$, and an argument similar to (4.5), we obtain that with probability 1

$$
\sum_{n=m}^{\infty}\left|b_{n} Y_{n}^{\prime \prime}\right|=O\left(m^{-(\alpha-1 / 2)}(\log m)^{1 / 2}\right)
$$

Note that $E Y_{n}^{\prime}=0$ and $\sup _{n} E\left(Y_{n}^{\prime}\right)^{2}<\infty$. Therefore using the exponential bound (4.9) and an argument similar to that in Theorem 7 (see (4.10)-(4.13)), we can apply the Salem-Zygmund bound in Lemma 7 to show that

$$
P\left[\max _{t}\left|\sum_{2^{k}<n<2^{k+1}} b_{n} Y_{n}^{\prime} \sin \left(n t+\Phi_{n}\right)\right|>C\left\{2^{-k(2 \alpha-1)}\left(\log 2^{k+1}\right)\right\}^{1 / 2}\right]=O\left(2^{-k / 4}\right),
$$

where $C$ is a sufficiently large positive constant. Hence by the Borel-Cantelli lemma, it follows that with probability 1

$$
\sup _{t}\left|\sum_{2^{k}<n} b_{n} Y_{n}^{\prime} \sin \left(n t+\Phi_{n}\right)\right|=O\left(k^{1 / 2} 2^{-k(\alpha-1 / 2)}\right)
$$

From (4.44), (4.45), and (4.46), the desired conclusion (4.42) follows. 
LEMMA 11. Let $\frac{1}{2}<\alpha<\frac{3}{2}$ and let $\left\{b_{n}\right\}$ be a sequence of constants satisfying (4.21). Let $\gamma$ be a real constant. Then

$$
\begin{aligned}
b_{n} & =O\left(n^{-\alpha}\right), \\
\sum_{n=2}^{m} n^{3 / 2}\left|b_{n}-b_{n+1}\right|(\log n)^{-\gamma} & =O\left(m^{3 / 2-\alpha}(\log m)^{-\gamma}\right), \quad \text { and } \\
\sum_{n=m}^{\infty} n^{1 / 2}\left|b_{n}-b_{n+1}\right|(\log n)^{-\gamma} & =O\left(m^{-(\alpha-1 / 2)}(\log m)^{-\gamma}\right) .
\end{aligned}
$$

Proof. Let $A_{n}=\sum_{k=n}^{\infty}\left|b_{k}-b_{k+1}\right|$. Since $\left|b_{n}\right|<A_{n}$, (4.47) follows. Since

$$
n^{\lambda}(\log n)^{-\gamma}-(n-1)^{\lambda}(\log (n-1))^{-\gamma} \sim \lambda n^{\lambda-1}(\log n)^{-\gamma}
$$

for $\lambda=\frac{1}{2}$ or $\frac{3}{2}$, and since $A_{n}=O\left(n^{-\alpha}\right)$, we obtain (4.48) and (4.49) by using summation by parts.

Proof of TheORem 7. Since $p>(6 \alpha+1) /(2 \alpha-1)$, we can choose $\eta$ and $\gamma$ such that

$$
\eta\left(\alpha-\frac{1}{2}\right)>\frac{1}{2}, \quad \gamma>\eta\left(\frac{3}{2}-\alpha\right)
$$

and $p>4(1+\gamma)+1$. By Lemma 9 , redefining the random variables on a new probability space if necessary, there exist independent normal random variables $\xi_{n}$ such that $E \xi_{n}=0, E \xi_{n}^{2}=\sigma_{n}^{2}$ and (4.38) holds. Let

$$
U(t)=\sum_{1}^{\infty} b_{n} \xi_{n} \sin (n t+\theta) .
$$

Then $E(U(t+s)-U(s))^{2}=v_{s}(t)$, where $v_{s}(t)$ is as defined in (4.20). We now show that $U(t)$ satisfies the condition (4.27) with $\beta=2 \alpha-1$.

By (4.19), $\sigma_{n}^{2}=O(1)$, and by Lemma $11, b_{n}=O\left(n^{-\alpha}\right)$. Moreover,

$$
\begin{aligned}
& \left|v_{s}(t+h)-v_{s}(h)\right| \\
& <\sum_{1}^{\infty} b_{n}^{2} \sigma_{n}^{2}|\sin (n t+n(h+s)+\theta)-\sin (n(h+s)+\theta)| \\
& \quad \times\{|\sin (n(t+h)+n s+\theta)-\sin (n s+\theta)| \\
& \quad+|\sin (n h+n s+\theta)-\sin (n s+\theta)|\} .
\end{aligned}
$$

First consider the case $|h|<|t|$ and $|t|>0$. Then by (4.3) and (4.52),

$$
\begin{aligned}
\left|v_{s}(t+h)-v_{s}(h)\right| & <8\left\{\sum_{n<|t|^{-1}} b_{n}^{2} \sigma_{n}^{2} n^{2}|t|(|t|+2|h|)+\sum_{n>|t|^{-1}} b_{n}^{2} \sigma_{n}^{2}\right\} \\
& <C|t|^{2 \alpha-1} \text { if }|h|<|t|<\delta,
\end{aligned}
$$

for some positive $\delta$ (sufficiently small) and $C$ (sufficiently large). Hence (4.27) holds in this case. Now consider the case $\alpha>1$ and $|h|>|t|>0$. Then by (4.3) and (4.52),

$$
\begin{aligned}
\mid v_{s}(t+ & h)-v_{s}(h) \mid \\
& <8\left\{3|t||h| \sum_{n<|h|^{-1}} n^{2} b_{n}^{2} \sigma_{n}^{2}+|t| \sum_{|h|^{-1}<n<|t|^{-1}} n b_{n}^{2} \sigma_{n}^{2}+\sum_{n>|t|^{-1}} b_{n}^{2} \sigma_{n}^{2}\right\} \\
& <C|t||h|^{2 \alpha-2} \text { if } 0<|t|<|h|<\delta .
\end{aligned}
$$


Since $1>(2 \alpha-1) / 2,(4.27)$ still holds. For the case $\alpha=1$ and $|h|>|t|>0$, take $\frac{1}{2}<\gamma<1$ and note that by (4.3) and (4.52),

$$
\begin{aligned}
\left|v_{s}(t+h)-v_{s}(h)\right| & <C\left\{|t||h| \sum_{n<|h|^{-1}} 1+\sum_{|h|^{-1}<n<|t|^{-1}}(n|t|)^{\gamma} n^{-2}+\sum_{n>|t|^{-1}} n^{-2}\right\} \\
& <3(1-\gamma)^{-1} C|t|^{\gamma}|h|^{1-\gamma} \text { if } 0<|t|<|h|<\delta .
\end{aligned}
$$

Finally, for the case $\alpha<1$, we obtain by (4.3) and (4.52) that

$$
\begin{aligned}
\left|v_{s}(t+h)-v_{s}(h)\right| & <C\left\{|t| \sum_{n<|t|^{-1}} n^{1-2 \alpha}+\sum_{n>|t|^{-1}} n^{-2 \alpha}\right\} \\
& <(1-\alpha)^{-1}\left(\alpha-\frac{1}{2}\right)^{-1} C|t|^{2 \alpha-1} \text { if } 0<|t|<\delta .
\end{aligned}
$$

Hence (4.27) again holds.

In view of (4.22) and (4.27), $U(t)$ satisfies the assumptions of Lemma 8 with $\beta=2 \alpha-1$. Hence by Lemma 8 , it suffices to show that for some $\rho>0$,

$$
\begin{aligned}
& Z(t)-Z(0)=U(t)-U(0)+R(t) \\
& \quad \text { with } R(t)=o\left(|t|^{\alpha-1 / 2} /\left(\log |t|^{-1}\right)^{\rho}\right) \text { a.s. }
\end{aligned}
$$

Let $S_{n}=\Sigma_{1}^{n} Y_{i}$ and $\hat{S}_{n}=S_{n}-\Sigma_{1}^{n} \xi_{i}$. By (4.38), with probability 1,

$$
\hat{S}_{n}=o\left(n^{1 / 2}(\log n)^{-\gamma}\right)
$$

Let $m=m(t)$ be the positive integer of the form $m=2^{k}$ defined by

$$
m=2^{k}<|t|^{-1}\left(\log |t|^{-1}\right)^{\eta}<2^{k+1} \text {. }
$$

By Lemma 10, with probability 1 ,

$$
\begin{array}{r}
\sup _{s}\left|\sum_{n=m+1}^{\infty} b_{n} Y_{n} \sin (n s+\theta)\right|+\sup _{s}\left|\sum_{n=m+1}^{\infty} b_{n} \xi_{n} \sin (n s+\theta)\right| \\
=O\left(k^{1 / 2} 2^{-k(\alpha-1 / 2)}\right)=O\left(|t|^{\alpha-1 / 2}\left(\log |t|^{-1}\right)^{-\eta(\alpha-1 / 2)+1 / 2}\right) .
\end{array}
$$

Let $\Delta_{n}(t)=\sin (n t+\theta)-\sin \theta$. Summation by parts gives

$$
\begin{aligned}
\sum_{n=1}^{m} b_{n} Y_{n}\{ & \sin (n t+\theta)-\sin \theta\} \\
= & b_{m} S_{m} \Delta_{m}(t)+\sum_{n=1}^{m-1}\left\{b_{n} \Delta_{n}(t)-b_{n+1} \Delta_{n+1}(t)\right\} S_{n} \\
= & \sum_{n=1}^{m} b_{n} \xi_{n}\{\sin (n t+\theta)-\sin \theta\}+b_{m} \hat{S}_{m} \Delta_{m}(t) \\
& +\sum_{n=1}^{m-1}\left\{b_{n} \Delta_{n}(t)-b_{n+1} \Delta_{n+1}(t)\right\} \hat{S}_{n} .
\end{aligned}
$$


Since $\left|\Delta_{n}(t)\right|<2|\sin (n t / 2)|$ and $\left|\Delta_{n}(t)-\Delta_{n+1}(t)\right|<2|\sin (t / 2)|$, we obtain that

$$
\begin{aligned}
\sum_{n=1}^{m-1}\left|b_{n} \Delta_{n}(t)-b_{n+1} \Delta_{n+1}(t)\right|\left|\hat{S}_{n}\right|< & 2 \sum_{n=1}^{m-1}\left|b_{n}-b_{n+1}\right||\sin (n t / 2)|\left|\hat{S}_{n}\right| \\
& +2|\sin (t / 2)| \sum_{n=1}^{m-1}\left|b_{n+1} \hat{S}_{n}\right|
\end{aligned}
$$

From (4.54), (4.55), and the fact that $b_{n}=O\left(n^{-\alpha}\right)$, it follows that with probability 1

$$
\begin{aligned}
b_{m} \hat{S}_{m} \Delta_{m}(t)=O\left(|t|^{\alpha-1 / 2}\left(\log |t|^{-1}\right)^{-\eta(\alpha-1 / 2)-\gamma}\right), \text { and } \\
\begin{aligned}
|t| \sum_{n=1}^{m-1}\left|b_{n+1} \hat{S}_{n}\right| & =O\left(|t| \sum_{n=2}^{m} n^{-\alpha+1 / 2}(\log n)^{-\gamma}\right) \\
& =O\left(|t|^{\alpha-1 / 2}\left(\log |t|^{-1}\right)^{\eta(3 / 2-\alpha)-\gamma}\right) .
\end{aligned}
\end{aligned}
$$

Moreover, by (4.3), (4.54), (4.55), and Lemma 11,

$$
\begin{aligned}
\sum_{n=1}^{\infty}\left|b_{n}-b_{n+1}\right||\sin (n t / 2)|\left|\hat{S}_{n}\right|= & O\left(|t| \sum_{2<n<|t|^{-1}} n^{3 / 2}\left|b_{n}-b_{n+1}\right|(\log n)^{-\gamma}\right) \\
& +O\left(\sum_{n>|t|^{-1}} n^{1 / 2}\left|b_{n}-b_{n+1}\right|(\log n)^{-\gamma}\right) \\
= & O\left(|t|^{\alpha-1 / 2}\left(\log |t|^{-1}\right)^{-\gamma}\right) .
\end{aligned}
$$

From (4.50) and (4.56)-(4.61), it follows that (4.53) holds for some $\rho>0$, and the proof is complete.

As an immediate application of Theorem 7, we obtain the following

COROLlary 4. With the same notations and assumptions as in Theorem 7, write $\log _{2} x=\log \log x$ and $\log _{k} x=\log \left(\log _{k-1} x\right)$ for $k>3$. Then for $k>5$,

$$
\begin{gathered}
P\left[|Z(t)-Z(0)|<v_{0}^{1 / 2}(t)\left\{2 \log _{2}|t|^{-1}+\frac{2 \alpha+1}{2 \alpha-1} \log _{3}|t|^{-1}\right.\right. \\
\left.+\cdots+2 \log _{k-1}|t|^{-1}+(2+\delta) \log _{k}|t|^{-1}\right\}^{1 / 2} \\
=1 \text { or } 0 \text { according as } \delta>1 \text { or } \delta<1 .
\end{gathered}
$$

We now apply Corollary 4 to give the following

Proof of Theorem 2. Since $a_{n} \downarrow, \sum_{k=n}^{\infty}\left|a_{k}-a_{k+1}\right|=a_{n}$ and therefore $\left\{a_{n}\right\}$ satisfies the condition (4.21). Without loss of generality we shall assume that $\sigma>0$. Then (4.18) holds. We now show that the condition (4.22) is satisfied by

$$
v_{s}(t)=\sigma^{2} \sum_{1}^{\infty} n^{-2 \alpha}\{\sin (n t+n s)-\sin n s\}^{2} \text {. }
$$


Note that for $t \neq 0$,

$$
v_{s}(t) \geqslant 4 \sigma^{2}\left(\sin \frac{\pi}{4}\right)^{2}\left(\frac{5 \pi}{8|t|}\right)^{-2 \alpha} \sum_{\pi / 4<n|t| / 2<5 \pi / 16} \cos ^{2}\left(\frac{1}{2} n t+n s\right) .
$$

For $s \neq 0$, let $P_{1}, \ldots, P_{m}$ be $m$ points placed consecutively (in the counterclockwise direction if $s>0$ and in the clockwise direction if $s<0$ ) on the unit circle such that the arc joining $P_{i}$ and $P_{i+1}$ has length $|s|$. Then as $m \rightarrow \infty$ and $s \rightarrow 0$, the number of points $P_{i}$ falling in a given arc of length $\pi / 16$ is asymptotic to $m / 32$, uniformly in the position of the initial point $P_{1}$. Hence in view of (4.62), there exists $\delta>0$ sufficiently small such that for $0<|t|<\delta$ and $0<|s|<\delta$,

$$
v_{s}(t) \geqslant\left\{\left(\frac{8|t|}{5 \pi}\right)^{2 \alpha} \sigma^{2} \cos ^{2}\left(\frac{5 \pi}{16}+\frac{\pi}{16}\right)\right\} \frac{1}{32}\left(\frac{\pi}{8|t|}\right) .
$$

Therefore (4.22) holds with $t \neq 0$ and $s \neq 0$. Clearly (4.22) also holds when $t=0$. The case $s=0$ is contained in

$$
v_{0}(t) \sim A_{\alpha} \sigma^{2}|t|^{2 \alpha-1} \text { as } t \rightarrow 0,
$$

where $A_{\alpha}$ is as defined by (1.9) with $A_{1}=\pi / 2$.

To prove (4.63), we note that for $0<a<b$,

$$
\begin{aligned}
|t|^{2 \alpha} \sum_{a|t|^{-1}<n<b|t|^{-1}}|n t|^{-2 \alpha} \sin ^{2} n t & \\
& \sim|t|^{2 \alpha-1} \int_{a}^{b} x^{-2 \alpha} \sin ^{2} x d x \quad \text { as }|t| \rightarrow 0+.
\end{aligned}
$$

Moreover, as $|t| \rightarrow 0+$,

$$
\sum_{n<a|t|^{-1}} n^{-2 \alpha} \sin ^{2} n t<t^{2} \sum_{n<a|t|^{-1}} n^{2-2 \alpha} \sim(3-2 \alpha)^{-1} a^{3-2 \alpha}|t|^{2 \alpha-1},
$$

and

$$
\sum_{n>b|t|^{-1}} n^{-2 \alpha} \sim(2 \alpha-1)^{-1}(|t| / b)^{2 \alpha-1}
$$

Therefore letting $a \downarrow 0$ and $b \rightarrow \infty$ in (4.64)-(4.66), we obtain

$$
\begin{aligned}
v_{0}(t) & \sim \sigma^{2}|t|^{2 \alpha-1} \int_{0}^{\infty} x^{-2 \alpha} \sin ^{2} x d x \\
& =(\pi / 2) \sigma^{2}|t|^{2 \alpha-1}, \quad \text { if } \alpha=1, \\
& =A_{\alpha} \sigma^{2}|t|^{2 \alpha-1}, \quad \text { if } \alpha \neq 1\left(\frac{1}{2}<\alpha<\frac{3}{2}\right)
\end{aligned}
$$

(cf. [3, p. 319]). Hence (4.63) follows. The desired conclusion (1.8) then follows from Corollary 4.

Acknowledgement. We wish to thank Professor Michael Marcus for his valuable suggestions. 


\section{REFERENCES}

1. L. Breiman, Probability, Addison-Wesley, Reading, Mass., 1968.

2. L. Carleson, On convergence and growth of partial sums of Fourier series, Acta Math. 116 (1966), 135-157.

3. A. Erdélyi, Tables of integral transforms, Vol. 1, McGraw Hill, New York, 1953.

4. G. H. Hardy and W. W. Rogonsinski, Notes on Fourier series. V: Summability ( $\left.R_{1}\right)$, Proc. Cambridge Philos. Soc. 45 (1949), 173-185.

5. G. A. Hunt, Random Fourier transforms, Trans. Amer. Math. Soc. 71 (1951), 38-69.

6. N. C. Jain, K. Jogdeo and W. F. Stout, Upper and lower functions for martingales and mixing processes, Ann. Probability 3 (1975), 119-145.

7. N. C. Jain and M. B. Marcus, Sufficient conditions for the continuity of stationary Gaussian processes and applications to random series of functions, Ann. Inst. Fourier (Grenoble) 24 (1974), 117-141.

8. Integrability of infinite sums of independent vector-valued random variables, Trans. Amer. Math. Soc. 212 (1975), 1-36.

9. J. P. Kahane, Propriétés locales des fonctions à séries de Fourier aléatories, Studia Math. 19 (1960), $1-25$.

10. Some random series of functions, Heath, Lexington, Mass., 1968.

11. M. Klass, Toward a universal law of the iterated logarithm, Part I, Z. Wahrscheinlichkeitstheorie und Verw. Gebiete 36 (1976), 165-178.

12. B. Kuttner, The relation between Riemann and Cesaro summability, Proc. London Math. Soc. 38 (1935), 273-283.

13. Some relations between different kinds of Riemann summability, Proc. London Math. Soc. 40 (1936), 524-540.

14. T. L. Lai, Summability methods for independent, identically distributed random variables, Proc. Amer. Math. Soc. 45 (1974), 253-261.

15. M. Loève, Probability theory, Van Nostrand, Princeton, N. J., 1962.

16. J. Marcinkiewicz and A. Zygmund, Sur les fonctions indépendantes, Fund. Math. 29 (1937), 60-90.

17. M. B. Marcus, A comparison of continuity conditions for Gaussian processes, Ann. Probability 1 (1973), 123-130.

18. __ Uniform convergence of random Fourier series, Ark. Mat. 13 (1975), 107-122.

19. Continuity and the central limit theorem for random trigonometric series, $\mathrm{Z}$. Wahrscheinlichkeitstheorie und Verw. Gebiete 42 (1978), 35-56.

20. M. B. Marcus and G. Pisier, Necessary and sufficient conditions for the uniform convergence of random trigonometric series, Lecture Notes Series No. 50, Aarhus Univ., Denmark, 1978.

21. _ Random Fourier series on locally compact Abelian groups, Séminaire de Probabilités (P. A. Meyer, Ed.), Lecture Notes in Math., vol. 709, Springer-Verlag, Berlin and New York, 1979, pp. $72-89$.

22. M. Nisio, On the extreme values of Gaussian processes, Osaka J. Math. 4 (1967), 313-326.

23. R. E. A. C. Paley and A. Zygmund, On some series of functions. I, II, III, Proc. Cambridge Philos. Soc. 26 (1930), 337-357; 26 (1930), 458-474; 28 (1932), 190-205.

24. C. Qualls and H. Watanabe, An asymptotic 0-1 behavior of Gaussian processes, Ann. Math. Statist. 42 (1971), 2029-2035.

25. R. Salem and A. Zygmund, Some properties of trigonometric series whose terms have random signs, Acta Math. 91 (1954), 245-301.

26. T. Sirao and H. Watanabe, On the upper and lower class for stationary Gaussian processes, Trans. Amer. Math. Soc. 147 (1970), 301-331.

27. V. Strassen, Almost sure behavior of sums of independent random variables and martingales, Proc. Fifth Berkeley Sympos. Math. Statist. Probability, Vol. 2, Univ. of California Press, Berkeley, Calif., 1965, pp. 315-343.

28. A. Zygmund, Trigonometric series. Vol. 1, Cambridge Univ. Press, Cambridge, Mass., 1959.

Department of Mathematical Statistics, Columbia University, New York, New York 10027 